IPMU-13-0143

KEK-TH-1641

\title{
Flavor of Gluino Decay in High-Scale Supersymmetry
}

\author{
Ryosuke Sato ${ }^{1}$, Satoshi Shirai ${ }^{2}$ and Kohsaku Tobioka ${ }^{3}$ \\ 1 Institute of Particle and Nuclear Studies, \\ High Energy Accelerator Research Organization (KEK) \\ Tsukuba 305-0801, Japan \\ ${ }^{2}$ Berkeley Center for Theoretical Physics, Department of Physics, \\ and Theoretical Physics Group, Lawrence Berkeley National Laboratory, \\ University of California, Berkeley, CA 94720, USA \\ ${ }^{3}$ Department of Physics, University of Tokyo, Tokyo 113-0033, Japan, \\ and Kavli Institute for the Physics and Mathematics of the Universe (WPI), \\ Todai Institutes for Advanced Study, University of Tokyo, \\ Kashiwa 277-8583, Japan
}

\begin{abstract}
The discovery of the Higgs boson with a mass of about $125 \mathrm{GeV}$ intimates us a possibility of a high-scale supersymmetry (SUSY) breaking model, where a sfermion mass scale is much higher than the electroweak scale. Although a general SUSY standard model can contribute to the low-energy flavor and/or CP-violating processes, the high-scale SUSY breaking model provides smaller signatures and therefore are less constrained, even in the presence of large flavor/CP violations of sfermions. However, a manner of gluino decay directly reflects the squark flavor structure and provides us a clue for the sfermion flavor structure. In this paper, we study the gluino decay in detail and discuss the interplay with the gluino decay and low-energy flavor and CP observation.
\end{abstract}




\section{Introduction}

A supersymmetric (SUSY) standard model (SSM) is a leading candidate of physics beyond the standard model (SM). However the most "natural" SUSY model suffers from longstanding problems, such as low-energy flavor/CP physics, direct sparticle search, Higgs boson mass, and cosmology. Many types of SSMs are studied to get over such difficulties.

The discovery of the $125 \mathrm{GeV}$ Higgs boson [1,2] indicates a possibility of a very simple framework of SSMs, i.e., high-scale SUSY scenarios [3, 4, 5, 6, 7]. The sfermion mass scale higher than $\mathcal{O}(10) \mathrm{TeV}$ can explain for the $125 \mathrm{GeV}$ Higgs boson [8, 9]. In addition, constraints of the SUSY flavor/CP problems and cosmological problems are greatly relaxed. As for the fermion sectors, the R-parity conservation sets gauginos and/or Higgsino mass scale to a $\mathrm{TeV}$ range to avoid the dark matter overabundance, which is also preferred by the gauge coupling unification. From a theoretical viewpoint, such a setup can be simply realized. For example, an assumption of some charge of the SUSY breaking field $X$ leads the sfermion mass $m_{\text {sfermion }} \gtrsim m_{3 / 2}$, keeping the gauginos light, $m_{\text {gaugino }} \sim 0.01 m_{3 / 2}$, which are generated via anomaly mediation effects [10]. By setting $m_{3 / 2}=\mathcal{O}\left(10^{2-3}\right) \mathrm{TeV}$, a viable SUSY mass spectrum can be obtained. After the discovery of the Higgs boson, such a framework gets more attention, variously called "Spread SUSY" [11], "Pure Gravity Mediation" [12], "Mini-Split" [13], "Simply Unnatural SUSY" [14] and so on. This framework predicts a Wino is the lightest SUSY particle (LSP). There are intensive studies of collider $[15,16]$ and dark matter $[17,18,19,20,21,22,23]$ signals of the Wino LSP.

Such a large mass hierarchy the between gaugino and scalar sector is not an uncommon feature of SUSY breaking models. This is because the mass of fermions can be protected from large radiative corrections thanks to some chiral symmetry and, on the other hand, that of the scalar particle is not protected, once SUSY is broken. For example, the large mass hierarchy also exists in some class of gauge mediated SUSY breaking (GMSB) models. See, e.g., Refs. [24, 25] for such models. This feature quite often comes from underlying symmetry of the model and/or stability conditions of the SUSY breaking vacua $[26,27]$. Motivated by the discovery of the Higgs boson, such GMSB models also have been studied $[13,28]$. 
Several kinds of SUSY breaking models realize the high-scale SUSY spectrum. Although they predict similar spectra at a TeV scale, the gravitino mass and sfermion sector have strong model dependence. One of the characteristic features of each model appears in the sfermion sector. For example, without any flavor symmetry, we can expect flavor violating soft parameters of the order of the scalar mass scale. On the other hand, some flavor symmetry forbid such terms, depending on the symmetry charge assignments. Or a typical GMSB model predicts just the minimal flavor violation. A grand unified theory (GUT) may determine the mass relation among the sfermion sector. Therefore, probing the sfermion sector is essential to study the underlying model. The information on the sfermion sector is also important for us to discuss the prospects of precise measurements of flavor/CP experiments.

For the future flavor/CP observations and deeper understanding the underlying theory of the high-scale SUSY models, it is very important to know the property of the sfermion sector, such as its mass scale and flavor structure. Since the direct production of heavy sfermions is out of range of the LHC, we must consider indirect ways to probe the sfermion sector. A cosmological signature such as gravitational waves is an interesting possibility among them [29]. Another possibility is the gluino decay since it is sensitive to the squark sector. The most known feature of the gluino in the high-scale SUSY models is its longevity of the lifetime. Another interesting point is that the flavor violating decay modes of the gluino are allowed thanks to heavy squark masses satisfying flavor constraints for the SM particles. Hence, by studying the gluino decay in detail, we can get insights into the squark sectors. In this paper we study the gluino decay in the high-scale SUSY models.

For heavy squarks, quantum corrections to the gluino decay get large, and can significantly affect the gluino decay, compared to the tree-level calculation. Previously, there are some works to study the tree-level gluino decay with flavor/CP violation [30] and the quantum effect without flavor/CP violation in the high-scale SUSY models [31, 32]. In this study, we pay particular attention to the quantum effects on the flavor structure of the gluino decay and discuss the correlation with the low-energy flavor/CP violating rare processes. In section 2, we estimate the size of the quantum corrections for the gluino decay, using renormalization group equations. In section 3, we discuss the correlation 
between the low-energy flavor/CP observations and the gluino decay. In section 4, we briefly discuss the collider signatures. Section 5 is devoted to summary and discussions.

\section{Gluino Decay in High-scale SUSY Models}

In the high-scale SUSY models, we cannot directly probe the sfermions at the LHC. However, the gluino decay requires virtual squark exchanges and therefore it reflects the squark sector. The most significant feature of the high-scale SUSY model is the longevity of the gluino lifetime since, as the squark mass scale goes high, the lifetime of the gluino can be very long. Typically, its decay length is $c \tau_{\tilde{g}} \sim 1 \mathrm{~cm}\left(m_{\tilde{q}} / 1000 \mathrm{TeV}\right)^{4}\left(m_{\tilde{g}} / 1 \mathrm{TeV}\right)^{-5}$. Such a long lifetime plays a significant role not only in collier signals but also in cosmology. Cosmological constraints disfavor the gluino lifetime much longer than 1 sec [33].

Another important feature is that the decay products of the gluino carry information on details of the squark flavor structure. For instance, if the third family squarks are lighter than the other squarks, the final states of the gluino decay are rich in bottom and top quarks. Furthermore, with the flavor violating structure of the squarks, we may observe the flavor violating gluino decay. In this way, the squark sector is testable via the gluino decay. To extract information on the sfermion sector via observation of the gluino decay, we need to sophisticate a prediction of the gluino decay, including quantum corrections.

In this section, we calculate partial decay widths of the gluino by a renormalization group (RG) method. We study the RG evolution of Wilson coefficients that govern the gluino decay with general flavor/CP structure and see the effects on the gluino decay.

\subsection{Effective Theory and RG Equations of Wilson Coefficients}

We assume that sfermions, heavy Higgs bosons and Higgsinos have the masses of around $\tilde{m}>\mathcal{O}(10) \mathrm{TeV}$ and that the size of $A$-terms is negligible. The soft mass terms relevant to our study are

$\mathcal{L}_{\mathrm{soft}}=-\frac{1}{2}\left(m_{\tilde{B}} \tilde{B} \tilde{B}+m_{\tilde{W}} \tilde{W}^{A} \tilde{W}^{A}+m_{\tilde{g}} \tilde{g}^{a} \tilde{g}^{a}+\right.$ h.c. $)-\tilde{Q}_{L}^{*} m_{\tilde{Q}_{L}}^{2} \tilde{Q}_{L}-\tilde{u}_{R}^{c} m_{\tilde{u}_{R}}^{2} \tilde{u}_{R}^{c *}-\tilde{d}_{R}^{c} m_{\tilde{d}_{R}}^{2} \tilde{d}_{R}^{c *}$, 
where $m_{\tilde{B}}, m_{\tilde{W}}$ and $m_{\tilde{g}}$ are complex gaugino masses and $m_{\tilde{Q}_{L}}^{2}, m_{\tilde{u}_{R}}^{2}$ and $m_{\tilde{d}_{R}}^{2}$ are $3 \times 3$ Hermitian matrices whose components are $\mathcal{O}\left(\tilde{m}^{2}\right)$. In this section, we take weak basis in which $Q_{L, i}$ and $\tilde{Q}_{L, i}$ form an $S U(2)_{L}$ doublet for each $i$. Thanks to this notation, we can write effective interactions and RG equations for their Wilson coefficients as $S U(2)_{L}$ symmetric forms. For a detail, see the Appendix A.

Below the scale $\tilde{m}$, the dynamics of the particles is described by an effective theory which contains the SM particles and gauginos. The gluino will mainly decay into a lighter SUSY particle by emitting two quarks or a gluon. These decay modes are dominantly induced by the following dimension five and six effective interactions by integrating out the heavy squarks:

$$
\begin{aligned}
Q_{Q, i j}^{\tilde{B}} & =\left(\tilde{B}^{\dagger} \bar{\sigma}^{\mu} \tilde{g}^{a}\right)\left(Q_{L, i}^{\dagger} \bar{\sigma}_{\mu} T^{a} Q_{L, j}\right), \\
Q_{u, i j}^{\tilde{B}} & =\left(\tilde{B}^{\dagger} \bar{\sigma}^{\mu} \tilde{g}^{a}\right)\left(u_{R, i}^{c} \sigma_{\mu} T^{a} u_{R, j}^{c \dagger}\right), \\
Q_{d, i j}^{\tilde{B}} & =\left(\tilde{B}^{\dagger} \bar{\sigma}^{\mu} \tilde{g}^{a}\right)\left(d_{R, i}^{c} \sigma_{\mu} T^{a} d_{R, j}^{c \dagger}\right), \\
Q_{7}^{\tilde{B}} & =\left(\tilde{B} \bar{\sigma}^{\mu \nu} \tilde{g}^{a}\right) G_{\mu \nu}^{a}, \\
Q_{i j}^{\tilde{W}} & =\left(\tilde{W}^{A \dagger} \bar{\sigma}^{\mu} \tilde{g}^{a}\right)\left(Q_{L, i}^{\dagger} \bar{\sigma}_{\mu} T^{a} \tau^{A} Q_{L, j}\right) .
\end{aligned}
$$

Here, $i, j=1,2,3$ are flavor indices, $A=1,2,3$ is an $S U(2)_{L}$ adjoint index and $a=$ $1, \cdots, 8$ is an $S U(3)_{C}$ adjoint index.

The effective Lagrangian contains the following interaction terms:

$\mathcal{L}_{\text {eff. }}=\frac{1}{\tilde{m}^{2}}\left[\sum_{i=1}^{3} \sum_{j=1}^{3}\left(C_{Q, i j}^{\tilde{B}} Q_{Q, i j}^{\tilde{B}}+C_{u, i j}^{\tilde{B}} Q_{u, i j}^{\tilde{B}}+C_{d, i j}^{\tilde{B}} Q_{d, i j}^{\tilde{B}}+C_{i j}^{\tilde{W}} Q_{i j}^{\tilde{W}}\right)+C_{7}^{\tilde{B}} Q_{7}^{\tilde{B}}\right]+h . c .$.

\section{Boundary condition at the squark mass scale}

We have to match our effective theory with the MSSM at the squark mass scale $\tilde{m}$. The Wilson coefficients $C(\tilde{m})$ 's are obtained by integrating out the squarks. At the leading order, we obtain,

$$
\begin{aligned}
C_{Q, i j}^{\tilde{B}}(\tilde{m}) & =-\frac{g_{s} g^{\prime}}{6} \tilde{m}^{2}\left(m_{\tilde{Q}_{L}}^{2}\right)_{i j}^{-1}, \\
C_{u, i j}^{\tilde{B}}(\tilde{m}) & =\frac{2 g_{s} g^{\prime}}{3} \tilde{m}^{2}\left(m_{\tilde{u}_{R}}^{2}\right)_{j i}^{-1}, \\
C_{d, i j}^{\tilde{B}}(\tilde{m}) & =-\frac{g_{s} g^{\prime}}{3} \tilde{m}^{2}\left(m_{\tilde{d}_{R}}^{2}\right)_{j i}^{-1},
\end{aligned}
$$



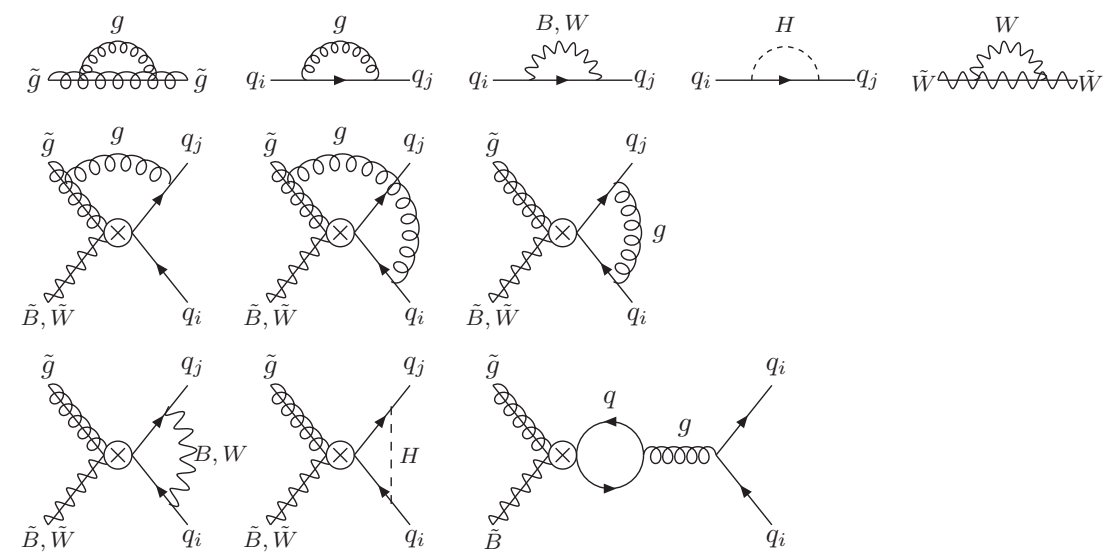

Figure 1: One-loop diagrams for the anomalous dimensions of quarks and gauginos (top) and the anomalous dimensions of operators and operator mixings (middle and bottom).

$$
\begin{aligned}
C_{7}^{\tilde{B}}(\tilde{m}) & =\frac{g_{s}^{2} g^{\prime}}{384 \pi^{2}}\left(m_{\tilde{g}}-m_{\tilde{B}}\right) \tilde{m}^{2}\left(\operatorname{tr}\left[\left(m_{\tilde{Q}_{L}}^{2}\right)^{-1}\right]-2 \operatorname{tr}\left[\left(m_{\tilde{u}_{R}}^{2}\right)^{-1}\right]+\operatorname{tr}\left[\left(m_{\tilde{d}_{R}}^{2}\right)^{-1}\right]\right), \\
C_{i j}^{\tilde{W}}(\tilde{m}) & =-\frac{g_{s} g}{2} \tilde{m}^{2}\left(m_{\tilde{Q}_{L}}^{2}\right)_{i j}^{-1} .
\end{aligned}
$$

Here, $m_{\tilde{Q}_{L}}^{2}, m_{\tilde{u}_{R}}^{2}$ and $m_{\tilde{d}_{R}}^{2}$ are the squark mass-squared matrices at the scale $\tilde{m}$. We treat the Wilson coefficients $C_{Q}^{\tilde{B}}, C_{u}^{\tilde{B}}, C_{d}^{\tilde{B}}$ and $C^{\tilde{W}}$ as $3 \times 3$ matrices in flavor space. In the high-scale SUSY models, there is a large hierarchy between the gaugino mass scale and the squark mass scale $\tilde{m}$. Therefore, the resummation of leading logarithm corrections becomes important.

\section{RG equations for Wilson coefficients}

Now, we describe the RG evolution of the Wilson coefficients at one-loop level. We treat SM-like Yukawa couplings $Y_{u}$ and $Y_{d}$ as $3 \times 3$ matrices in flavor space. They are defined as,

$$
\mathcal{L}_{\text {yukawa }}=H Q_{L} Y_{u} u_{R}^{c}+H^{*} Q_{L} Y_{d} d_{R}^{c}+\text { h.c.. }
$$

Here, $H$ is the SM-like Higgs doublet field. RG equations for the gauge coupling constants are given by,

$$
16 \pi^{2} \frac{d g^{\prime}}{d \log \mu}=\frac{41}{6} g^{\prime 3}, \quad 16 \pi^{2} \frac{d g}{d \log \mu}=-\frac{11}{6} g^{3}, \quad 16 \pi^{2} \frac{d g_{s}}{d \log \mu}=-5 g_{s}^{3} .
$$


For the quark Yukawa couplings,

$$
\begin{aligned}
16 \pi^{2} \frac{d Y_{u}}{d \log \mu} & =\left[\frac{3}{2} Y_{u} Y_{u}^{\dagger}-\frac{3}{2} Y_{d} Y_{d}^{\dagger}+3 \operatorname{tr}\left(Y_{u} Y_{u}^{\dagger}+Y_{d} Y_{d}^{\dagger}\right)-\frac{17}{12} g^{\prime 2}-\frac{9}{4} g_{2}^{2}-8 g_{s}^{2}\right] Y_{u}, \\
16 \pi^{2} \frac{d Y_{d}}{d \log \mu} & =\left[-\frac{3}{2} Y_{u} Y_{u}^{\dagger}+\frac{3}{2} Y_{d} Y_{d}^{\dagger}+3 \operatorname{tr}\left(Y_{u} Y_{u}^{\dagger}+Y_{d} Y_{d}^{\dagger}\right)-\frac{5}{12} g^{\prime 2}-\frac{9}{4} g_{2}^{2}-8 g_{s}^{2}\right] Y_{d} .
\end{aligned}
$$

Here, we neglect the lepton Yukawa interactions. The RG equations for $g^{\prime}, Y_{u}$ and $Y_{d}$ at one-loop level are same as the SM ones [34]. RG equations for the Wilson coefficients are given by, ${ }^{1}$

$$
\begin{aligned}
16 \pi^{2} \frac{d C_{Q}^{\tilde{B}}}{d \log \mu} & =\frac{1}{2}\left\{Y_{u} Y_{u}^{\dagger}+Y_{d} Y_{d}^{\dagger}, C_{Q}^{\tilde{B}}\right\}-Y_{u} C_{u}^{\tilde{B}} Y_{u}^{\dagger}-Y_{d} C_{d}^{\tilde{B}} Y_{d}^{\dagger}-3 g_{s}^{2} N_{C} C_{Q}^{\tilde{B}}+\frac{2}{3} g_{s}^{2} \hat{\mathbf{S}}, \\
16 \pi^{2} \frac{d C_{u}^{\tilde{B}}}{d \log \mu} & =\left\{Y_{u}^{\dagger} Y_{u}, C_{u}^{\tilde{B}}\right\}-2 Y_{u}^{\dagger} C_{Q}^{\tilde{B}} Y_{u}-3 g_{s}^{2} N_{C} C_{u}^{\tilde{B}}+\frac{2}{3} g_{s}^{2} \hat{\mathbf{S}}, \\
16 \pi^{2} \frac{d C_{d}^{\tilde{B}}}{d \log \mu} & =\left\{Y_{d}^{\dagger} Y_{d}, C_{d}^{\tilde{B}}\right\}-2 Y_{d}^{\dagger} C_{Q}^{\tilde{B}} Y_{d}-3 g_{s}^{2} N_{C} C_{d}^{\tilde{B}}+\frac{2}{3} g_{s}^{2} \hat{\mathbf{S}}, \\
16 \pi^{2} \frac{d C_{7}^{\tilde{B}}}{d \log \mu} & =\left(\frac{2}{3} N_{F}-6 N_{C}\right) g_{s}^{2} C_{7}^{\tilde{B}}, \\
16 \pi^{2} \frac{d C^{\tilde{W}}}{d \log \mu} & =\frac{1}{2}\left\{Y_{u} Y_{u}^{\dagger}+Y_{d} Y_{d}^{\dagger}, C^{\tilde{W}}\right\}-3 g_{s}^{2} N_{C} C^{\tilde{W}}-6 g^{2} C^{\tilde{W}} .
\end{aligned}
$$

Here, $\hat{\mathbf{S}}=\left(2 \operatorname{tr} C_{Q}^{\tilde{B}}+\operatorname{tr} C_{u}^{\tilde{B}}+\operatorname{tr} C_{d}^{\tilde{B}}\right) \mathbf{1},\{A, B\}=A B+B A, \mathbf{1}=\operatorname{diag}(1,1,1), N_{F}=6$ and $N_{C}=3$. Again, $C_{Q}^{\tilde{B}}, C_{u}^{\tilde{B}}, C_{d}^{\tilde{B}}$ and $C^{\tilde{W}}$ are $3 \times 3$ matrices in flavor space. We show one-loop diagrams which contribute to the above RG equations in Fig. 1. We have checked Eqs. (17-21) are consistent with Ref. [31] in the flavor-symmetric case. ${ }^{2}$ In the following of this section, we will discuss the flavor structures of the Wilson coefficients in more detail.

\subsection{Resummed Wilson Coefficient}

Here, we show an analytic solution for an approximated situation. We neglect the Yukawa couplings except for the top Yukawa, namely, we take the following Yukawa matrices,

$$
Y_{u}=\left(\begin{array}{ccc}
0 & 0 & 0 \\
0 & 0 & 0 \\
0 & 0 & y_{t}
\end{array}\right), \quad Y_{d}=\left(\begin{array}{ccc}
0 & 0 & 0 \\
0 & 0 & 0 \\
0 & 0 & 0
\end{array}\right) .
$$

\footnotetext{
${ }^{1}$ One may worry about mixing with other operators, such as, $d_{a b c}\left(\tilde{B}^{\dagger} \bar{\sigma}^{\mu} \tilde{g}^{a}\right)\left(\tilde{g}^{b \dagger} \bar{\sigma}_{\mu} \tilde{g}^{c}\right)$ and $d_{a b c}\left(\tilde{B} \tilde{g}^{a}\right)\left(\tilde{g}^{b} \tilde{g}^{c}\right)$. Here, $d_{a b c}=\operatorname{tr}\left[T^{a}\left\{T^{b}, T^{c}\right\}\right]$. However, $Q_{Q, i j}^{\tilde{B}}, Q_{u, i j}^{\tilde{B}}, Q_{d, i j}^{\tilde{B}}$ and $Q_{7}^{\tilde{B}} \operatorname{do}$ not mix with them at one-loop level.

${ }^{2}$ If the Higgsino is lighter than the gluino, we have to consider effective operators which include Higgsinos. For RG equation of their Wilson coefficients, see the Appendix B.
} 
To write down the solution to the RG equations, we define the following variables: ${ }^{3}$

$$
\begin{aligned}
\eta_{1} & \equiv \frac{\alpha^{\prime}(\tilde{m})}{\alpha^{\prime}(\mu)}=1-\frac{41 \alpha^{\prime}(\tilde{m})}{12 \pi} \log \frac{\mu}{\tilde{m}}=\left(1+\frac{41 \alpha^{\prime}(\mu)}{12 \pi} \log \frac{\mu}{\tilde{m}}\right)^{-1} \\
\eta_{2} & \equiv \frac{\alpha(\tilde{m})}{\alpha(\mu)}=1+\frac{11 \alpha(\tilde{m})}{12 \pi} \log \frac{\mu}{\tilde{m}}=\left(1-\frac{11 \alpha(\mu)}{12 \pi} \log \frac{\mu}{\tilde{m}}\right)^{-1} \\
\eta_{3} & \equiv \frac{\alpha_{s}(\tilde{m})}{\alpha_{s}(\mu)}=1+\frac{5 \alpha_{s}(\tilde{m})}{2 \pi} \log \frac{\mu}{\tilde{m}}=\left(1-\frac{5 \alpha_{s}(\mu)}{2 \pi} \log \frac{\mu}{\tilde{m}}\right)^{-1} \\
\eta_{t} & \equiv \frac{\alpha_{t}(\tilde{m})}{\alpha_{t}(\mu)}, \\
\xi_{t} & \equiv \exp \left(-\int_{\mu}^{\tilde{m}} \frac{y_{t}^{2}\left(\mu^{\prime}\right)}{16 \pi^{2}} \frac{d \mu^{\prime}}{\mu^{\prime}}\right)=\eta_{t}^{-1 / 9} \eta_{1}^{-17 / 738} \eta_{2}^{3 / 22} \eta_{3}^{8 / 45} \\
y & \equiv \eta_{3}^{4 / 5}-1, \\
z & \equiv \frac{1}{3}\left(\xi_{t}^{3}-1\right), \\
z^{\prime} & \equiv \frac{1}{3}\left(\xi_{t}^{3 / 2}-1\right) \\
\bar{C}^{\tilde{B}}(\tilde{m}) & \equiv \frac{1}{12}\left(2 \operatorname{tr} C_{Q}^{\tilde{B}}(\tilde{m})+\operatorname{tr} C_{u}^{\tilde{B}}(\tilde{m})+\operatorname{tr} C_{d}^{\tilde{B}}(\tilde{m})\right)
\end{aligned}
$$

We show $\eta_{1}, \eta_{2}, \eta_{3}, \eta_{t}$ and $\xi_{t}$ as functions of $\tilde{m}$ with $\mu=\left|m_{\tilde{g}}\right|$ in Fig. 2. Here we set $m_{\tilde{g}}=1.5 \mathrm{TeV}, m_{\tilde{W}}=200 \mathrm{GeV}$ and $m_{\tilde{B}}=400 \mathrm{GeV} \cdot{ }^{4}$ By using these variables given in Eqs. (24-32), the solution to the RG equations in Eqs. (17-21) is written as,

$$
\begin{aligned}
C_{i j}^{\tilde{W}}(\mu) & =\eta_{3}^{-9 / 10} \eta_{2}^{-18 / 11} C_{i j}^{\tilde{W}}(\tilde{m}), \\
C_{3 i}^{\tilde{W}}(\mu) & =\eta_{3}^{-9 / 10} \eta_{2}^{-18 / 11} \xi_{t}^{1 / 2} C_{3 i}^{\tilde{W}}(\tilde{m}), \\
C_{33}^{\tilde{W}}(\mu) & =\eta_{3}^{-9 / 10} \eta_{2}^{-18 / 11} \xi_{t} C_{33}^{\tilde{W}}(\tilde{m}), \\
C_{Q, i i}^{\tilde{B}}(\mu) & =\eta_{3}^{-9 / 10}\left[C_{Q, i i}^{\tilde{B}}(\tilde{m})+y \bar{C}^{\tilde{B}}(\tilde{m})\right], \\
C_{Q, 12}^{\tilde{B}}(\mu) & =\eta_{3}^{-9 / 10} C_{Q, 12}^{\tilde{B}}(\tilde{m}),
\end{aligned}
$$

${ }^{3}$ In the limit of $g^{\prime}=g=0$, we can get analytic form of $\eta_{t}$ :

$$
\eta_{t}=\eta_{3}^{8 / 5}-\frac{3}{2} \frac{\alpha_{t}(\tilde{m})}{\alpha_{s}(\tilde{m})}\left(\eta_{3}^{8 / 5}-\eta_{3}\right)=\left(\eta_{3}^{-8 / 5}-\frac{3}{2} \frac{\alpha_{t}(\mu)}{\alpha_{s}(\mu)}\left(\eta_{3}^{-8 / 5}-\eta_{3}^{-1}\right)\right)^{-1} .
$$

${ }^{4}$ Very recently, a search of disappearing charged tracks by the ATLAS collaboration gives severe constraint on the mass of the Wino LSP. If the Higgsino mass is large enough and we adopt two-loop level mass splitting between $\tilde{W}^{ \pm}$and $\tilde{W}^{0}$ [35], we obtain $m_{\tilde{W}}>270 \mathrm{GeV}$ [36]. However, our analysis is almost independent on the Wino mass, then, we take this value as a reference point. 


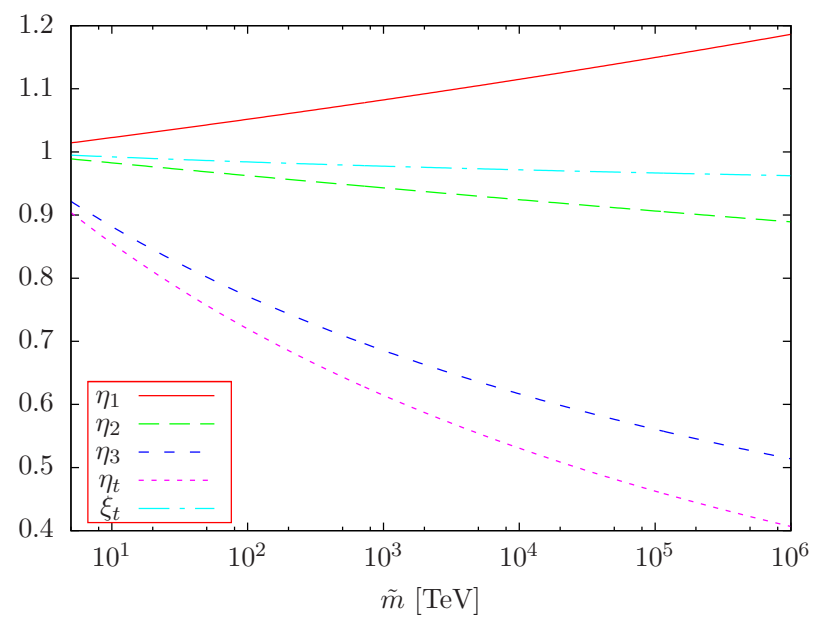

Figure 2: Variables $\eta_{1}, \eta_{2}, \eta_{3}, \eta_{t}$ and $\xi_{t}$ in Eqs. (24-28) as functions of $\tilde{m}$, fixing $\mu=\left|m_{\tilde{g}}\right|$. We take $m_{\tilde{g}}=1.5 \mathrm{TeV}$ and $m_{\tilde{W}}=200 \mathrm{GeV}$.

$$
\begin{aligned}
C_{Q, 33}^{\tilde{B}}(\mu) & =\eta_{3}^{-9 / 10}\left[(1+z) C_{Q, 33}^{\tilde{B}}(\tilde{m})-z C_{u, 33}^{\tilde{B}}(\tilde{m})+y \bar{C}^{\tilde{B}}(\tilde{m})\right] \\
C_{Q, 3 i}^{\tilde{B}}(\mu) & =\eta_{3}^{-9 / 10}\left[\left(1+z^{\prime}\right) C_{Q, 3 i}^{\tilde{B}}(\tilde{m})-z^{\prime} C_{u, 3 i}^{\tilde{B}}(\tilde{m})\right] \\
C_{u, i i}^{\tilde{B}}(\mu) & =\eta_{3}^{-9 / 10}\left[C_{u, i i}^{\tilde{B}}(\tilde{m})+y \bar{C}^{\tilde{B}}(\tilde{m})\right] \\
C_{u, 12}^{\tilde{B}}(\mu) & =\eta_{3}^{-9 / 10} C_{u, 12}^{\tilde{B}}(\tilde{m}) \\
C_{u, 33}^{\tilde{B}}(\mu) & =\eta_{3}^{-9 / 10}\left[(1+2 z) C_{u, 33}^{\tilde{B}}(\tilde{m})-2 z C_{Q, 33}^{\tilde{B}}(\tilde{m})+y \bar{C}^{\tilde{B}}(\tilde{m})\right] \\
C_{u, 3 i}^{\tilde{B}}(\mu) & =\eta_{3}^{-9 / 10}\left[\left(1+2 z^{\prime}\right) C_{u, 3 i}^{\tilde{B}}(\tilde{m})-2 z^{\prime} C_{Q, 3 i}^{\tilde{B}}(\tilde{m})\right] \\
C_{d, i i}^{\tilde{B}}(\mu) & =\eta_{3}^{-9 / 10}\left[C_{d, i i}^{\tilde{B}}(\tilde{m})+y \bar{C}^{\tilde{B}}(\tilde{m})\right] \\
C_{d, 12}^{\tilde{B}}(\mu) & =\eta_{3}^{-9 / 10} C_{d, 12}^{\tilde{B}}(\tilde{m}) \\
C_{d, 33}^{\tilde{B}}(\mu) & =\eta_{3}^{-9 / 10}\left[C_{d, 33}^{\tilde{B}}(\tilde{m})+y \bar{C}^{\tilde{B}}(\tilde{m})\right] \\
C_{d, 3 i}^{\tilde{B}}(\mu) & =\eta_{3}^{-9 / 10} C_{d, 3 i}^{\tilde{B}}(\tilde{m}) \\
C_{7}^{\tilde{B}}(\mu) & =\eta_{3}^{-7 / 5} C_{7}^{\tilde{B}}(\tilde{m}) .
\end{aligned}
$$

Here, $i$ runs a light flavor index 1,2. Note that we have dropped off-diagonal elements of Yukawa matrices. Then, off-diagonal elements of $C$ 's are valid only if the contribution of off-diagonal elements of Yukawa is much smaller than them. For example, $C_{12}^{\tilde{W}}(\mu)$ in Eq. 
(33) is valid only if $C_{12}^{\tilde{W}}(\tilde{m})$ is much larger than $Y_{u, 12} Y_{u, 22} C_{22}^{\tilde{W}}(\tilde{m})$ and $Y_{u, 13} Y_{u, 23} C_{33}^{\tilde{W}}(\tilde{m})$.

\subsection{Numerical Analysis of Gluino decay}

So far, we discussed leading logarithm corrections for the Wilson coefficients relevant to the gluino decay. To extract information on the squark sector from the gluino decay, it is important to take into account such corrections. In this subsection, we discuss the effects of leading logarithm corrections on the lifetime and decay pattern of the gluino numerically. In the present situation, gluino two-body decay $(\tilde{g} \rightarrow g \tilde{B})$ can be neglected because of one-loop suppression. ${ }^{5}$ In the limit that $\left|m_{\tilde{g}}\right|-\left|m_{\tilde{W}}\right|,\left|m_{\tilde{g}}\right|-\left|m_{\tilde{B}}\right| \gg m_{t}$ and $V_{\mathrm{CKM}} \simeq \mathbf{1}$, we can approximately get the following analytical formulae $[31,37]$ :

$$
\begin{aligned}
& \Gamma\left(\tilde{g} \rightarrow \tilde{B} u_{L i} \bar{u}_{L j}\right)= \Gamma\left(\tilde{g} \rightarrow \tilde{B} d_{L i} \bar{d}_{L j}\right)=\frac{\left|C_{Q, i j}^{\tilde{B}}\right|^{2}\left|m_{\tilde{g}}\right|^{5}}{1536 \pi^{3} \tilde{m}^{4}} f\left(m_{\tilde{B}} / m_{\tilde{g}}\right), \\
& \Gamma\left(\tilde{g} \rightarrow \tilde{B} u_{R i} \bar{u}_{R j}\right)=\frac{\left|C_{u, i j}^{\tilde{B}}\right|^{2}\left|m_{\tilde{g}}\right|^{5}}{1536 \pi^{3} \tilde{m}^{4}} f\left(m_{\tilde{B}} / m_{\tilde{g}}\right), \\
& \Gamma\left(\tilde{g} \rightarrow \tilde{B} d_{R i} \bar{d}_{R j}\right)=\frac{\left|C_{d, i j}^{\tilde{B}}\right|^{2}\left|m_{\tilde{g}}\right|^{5}}{1536 \pi^{3} \tilde{m}^{4}} f\left(m_{\tilde{B}} / m_{\tilde{g}}\right), \\
& \Gamma\left(\tilde{g} \rightarrow \tilde{W}^{0} u_{L i} \bar{u}_{L j}\right)=\Gamma\left(\tilde{g} \rightarrow \tilde{W}^{0} d_{L i} \bar{d}_{L j}\right)=\frac{\left|C_{i j}^{\tilde{W}}\right|^{2}\left|m_{\tilde{g}}\right|^{5}}{1536 \pi^{3} \tilde{m}^{4}} f\left(m_{\tilde{W}} / m_{\tilde{g}}\right), \\
& \Gamma\left(\tilde{g} \rightarrow \tilde{W}^{+} d_{L i} \bar{u}_{L j}\right)=\Gamma\left(\tilde{g} \rightarrow \tilde{W}^{-} u_{L i} \bar{d}_{L j}\right)=\frac{2\left|C_{i j}^{\tilde{W}}\right|^{2}\left|m_{\tilde{g}}\right|^{5}}{1536 \pi^{3} \tilde{m}^{4}} f\left(m_{\tilde{W}} / m_{\tilde{g}}\right) .
\end{aligned}
$$

Here, $f(x)=1-8|x|^{2}-12|x|^{4} \log |x|^{2}+8|x|^{6}-|x|^{8}+2\left(1+9|x|^{2}+6|x|^{4} \log |x|^{2}-9|x|^{4}+\right.$ $\left.6|x|^{4} \log |x|^{2}-|x|^{6}\right) \operatorname{Re}(x)$. As for operators whose dimension is higher than six, we use tree-level amplitudes in our numerical calculation. In the following of this section, we denote decay widths calculated by $C(\mu=\tilde{m})$ and $C\left(\mu=\left|m_{\tilde{g}}\right|\right)$ as "tree" and "resum", respectively.

\section{Total decay width}

It is pointed out that the lifetime of the gluino is significantly affected by the resummation of leading logarithmic corrections [31]. We show the lifetime of the gluino in Fig. 3. Here we take $m_{\tilde{Q}_{L}}^{2}=m_{\tilde{u}_{R}}^{2}=m_{\tilde{d}_{R}}^{2}=\tilde{m}^{2} \times \mathbf{1}$. We can see that the quantum corrections make the decay rate double for $\tilde{m}=10^{3} \mathrm{TeV}$.

\footnotetext{
${ }^{5}$ If the Higgsino is lighter than the gluino, the decay width of this two body decay mode is enhanced because of large logarithmic corrections [30, 31, 32]. Then, this mode can be significant.
} 


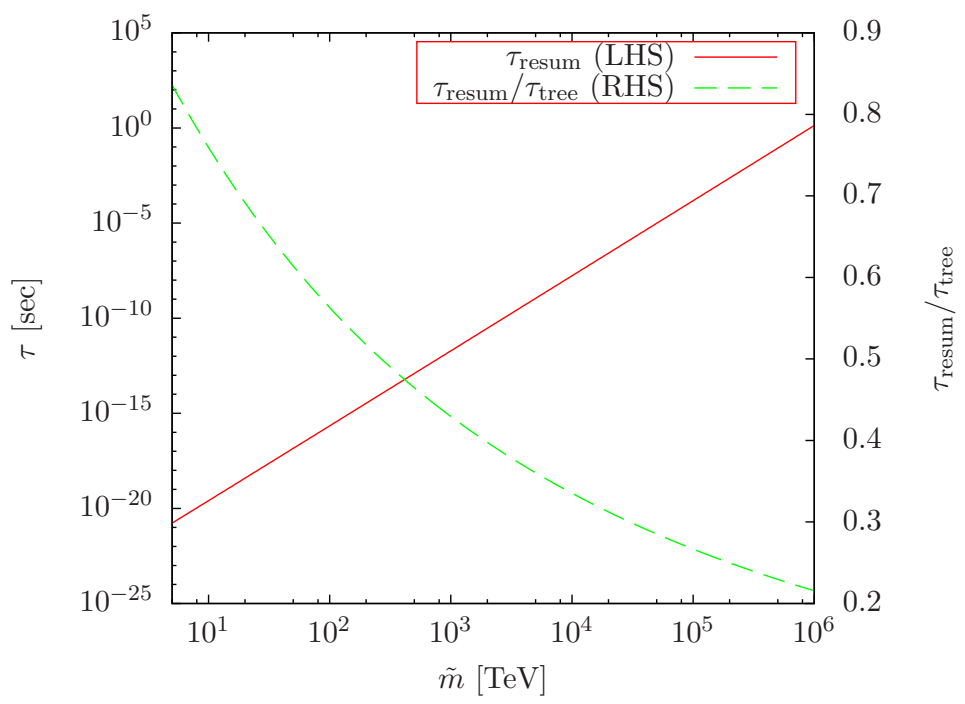

Figure 3: The red and solid line shows the lifetime of the gluino with resummation. The green and dashed line shows the ratio of the resummed lifetime to tree-level one. We take the gaugino masses as $m_{\tilde{W}}=200 \mathrm{GeV}, m_{\tilde{B}}=400 \mathrm{GeV}$ and $m_{\tilde{g}}=-1.5 \mathrm{TeV}$. For the squark mass, we take universal squark masses at the squark mass scale.

\section{Branching fraction of $\tilde{g} \rightarrow \tilde{W} q \bar{q}$ and $\tilde{g} \rightarrow \tilde{B} q \bar{q}$}

In the case of the heavy Higgsino, the low-mass neutralinos and charginos are almost pure Wino or Bino states. Therefore discriminating the gluino decay mode into a Wino and Bino can be experimentally viable. This branching ratio reveals the relation between left-handed squarks and right-handed ones. Thus, precise estimation of the branching ratio is significant.

At tree level, the Wilson coefficients of the operators involving $\tilde{W}$ are proportional to the gauge coupling $g$, and those involving $\tilde{B}$ are proportional to $g^{\prime}$. They are proportional to $\alpha^{\prime}$ and $\alpha$ at tree level, however, leading log resummation alters ratio between them.

In Fig. 4, we plot $R_{\tilde{B} / \tilde{W}}$ as a function of squark masses, which is defined as,

$$
R_{\tilde{B} / \tilde{W}} \equiv \frac{\sum_{q} \Gamma(\tilde{g} \rightarrow \tilde{B} q \bar{q})}{\sum_{q} \Gamma(\tilde{g} \rightarrow \tilde{W} q \bar{q})} .
$$

Here we take $m_{\tilde{Q}_{L}}^{2}=m_{\tilde{u}_{R}}^{2}=m_{\tilde{d}_{R}}^{2}=\tilde{m}^{2} \times \mathbf{1}$. We can see that resummation of leading logarithm corrections alters branching fractions by about $20 \%$ for $\tilde{m}=10^{3} \mathrm{TeV}$. 


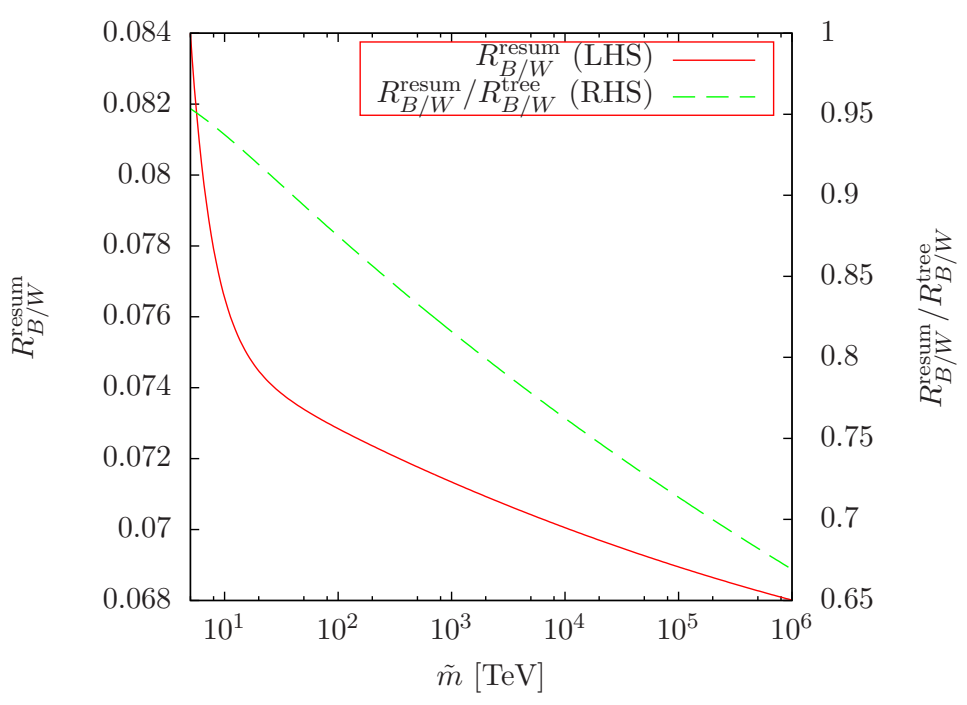

Figure 4: $\left.R_{\tilde{B} / \tilde{W}}\right|_{\text {resum }}$ (red and solid line) and the ratio of the resummed one to treelevel one (green and dashed line). We take the gaugino masses as $m_{\tilde{W}}=200 \mathrm{GeV}$, $m_{\tilde{B}}=400 \mathrm{GeV}$ and $m_{\tilde{g}}=-1.5 \mathrm{TeV}$. For the squark mass, we take universal squark mass at for $\tilde{m}=10^{3} \mathrm{TeV}$.quark mass scale.

\section{Flavor structure of $\tilde{g} \rightarrow \tilde{W} q \bar{q}$}

The flavor structure of $\tilde{g} \rightarrow \tilde{W} q \bar{q}$ is determined by $\left(m_{\tilde{Q}_{L}}^{2}\right)^{-1}$ at tree level, and it suffers from leading logarithm corrections because of the anomalous dimension of the quarks. An important source of the corrections is top Yukawa $y_{t}$. It changes $\tilde{g} \rightarrow \tilde{W}^{0} t \bar{t}$ relative ratio to $\tilde{g} \rightarrow \tilde{W} q \bar{q}$.

$$
R_{t t / q q}^{\tilde{W}} \equiv \frac{\Gamma\left(\tilde{g} \rightarrow \tilde{W}^{0} t \bar{t}\right)}{\Gamma\left(\tilde{g} \rightarrow \tilde{W} q_{i} \bar{q}_{j}\right)}
$$

where $i, j=1,2$. The ratio of resummed result to tree-level one is $\left(\left.R_{t t / q q}^{\tilde{W}}\right|_{\text {resum }}\right) /\left(\left.R_{t t / q q}^{\tilde{W}}\right|_{\text {tree }}\right)=$ $\xi_{t}^{2}$. As seen in Fig. 2, $R_{t t / q q}^{\tilde{W}}$ is decreased by the effect of resummation of leading logarithm correction by 3-4\%, compared to the tree-level result.

\section{Flavor structure of $\tilde{g} \rightarrow \tilde{B} q \bar{q}$}

Here, we discuss radiative correction for the following quantities:

$$
R_{t c / t t}^{\tilde{B}}=\frac{\Gamma(\tilde{g} \rightarrow \tilde{B} t \bar{c} / \tilde{B} \bar{t} c)}{\Gamma(\tilde{g} \rightarrow \tilde{B} t \bar{t})}, \quad R_{t c / c c}^{\tilde{B}}=\frac{\Gamma(\tilde{g} \rightarrow \tilde{B} t \bar{c} / \tilde{B} \bar{t} c)}{\Gamma(\tilde{g} \rightarrow \tilde{B} c \bar{c})} .
$$


Compared to the Wino case, radiative corrections for the decay into the Bino more significantly affect the flavor structure of the gluino decay. This is because the contribution of the top Yukawa coupling is larger. In addition, the penguin-like diagram contribution in Fig. 1 affects only the flavor diagonal part. Thus, the RG evolution of the Wilson coefficients has different feature between the flavor conserving and violating parts. We show numerical results in the following two sample cases.

- light $\tilde{u}_{R}$

In this case, we assume that the lightest squark $\tilde{q}_{R 1}$ is a mixture of $\tilde{t}_{R}$ and $\tilde{c}_{R}$, and other squarks are much heavier than them. The mixing angle $\theta_{u}$ is defined as $\tilde{q}_{R 1}=\cos \theta_{u} \tilde{t}_{R}+\sin \theta_{u} \tilde{c}_{R}$. Fig. 5 shows the numerical result of the gluino decay length, $R_{t c / t t}^{\tilde{B}}$ and $R_{t c / c c}^{\tilde{B}}$. Here we define a normalization variable $R$ for the gluino decay length as

$$
c \tau_{\tilde{g}}=R \times 1 \mathrm{~cm}\left(\frac{m_{\tilde{q}_{R 1}}}{1000 \mathrm{TeV}}\right)^{4} .
$$

We can see $\left.R_{t c / t t}^{\tilde{B}}\right|_{\text {resum }}\left(\left.R_{t c / c c}^{\tilde{B}}\right|_{\text {resum }}\right)$ is enhanced compared to $\left.R_{t c / t t}^{\tilde{B}}\right|_{\text {tree }}\left(\left.R_{t c / c c}^{\tilde{B}}\right|_{\text {tree }}\right)$ results by $10-20 \%$.

- mSUGRA-like case

In this case, at the "GUT" scale $\mu=2 \times 10^{16} \mathrm{GeV}$, we take the diagonal elements of the squark mass-squared as $m_{\tilde{Q}_{L}, i i}^{2}=m_{\tilde{u}_{R}, i i}^{2}=m_{\tilde{d}_{R}, i i}^{2}=m_{0}^{2}(i=1,2,3)$, the offdiagonal elements as $m_{\tilde{u}_{R}, 23}^{2}=m_{\tilde{u}_{R}, 32}^{2}=m_{23}^{2}$ and the other components to be zero. For other parameters, we take $m_{\tilde{W}}=200 \mathrm{GeV}, m_{\tilde{B}}=400 \mathrm{GeV}, m_{\tilde{g}}=-1.5 \mathrm{TeV}$ and $\tan \beta=1,3$. By using $R G$ equations for the squark soft parameters, we obtain physical squark masses and mixings. In calculation of soft mass RG evolution, we neglect terms other than top Yukawa parts. We also assume $b, \mu$ and $m_{H_{d}}^{2}$ are optimized so that the correct electroweak breaking occurs.

For estimation of the gluino decay width, we take the matching scale $\tilde{m}$ as the lightest squark mass. In this case, the RG effect from the top Yukawa coupling decreases $m_{\tilde{t}_{R}}$ and the decay into $t \bar{t} \tilde{B}$ is enhanced. Fig. 6 shows the numerical result of $R_{t c / t t}^{\tilde{B}}$. The shaded region shows the region where the lightest squark is lighter than the gluino or a tachyon. We can see $\left.R_{t c / t t}^{\tilde{B}}\right|_{\text {resum }}\left(\left.R_{t c / c c}^{\tilde{B}}\right|_{\text {resum }}\right)$ is enhanced compared to $\left.R_{t c / t t}^{\tilde{B}}\right|_{\text {tree }}$ $\left(R_{\text {tc } / \text { cc }}^{\tilde{B}} \mid\right.$ tree $)$ results by about $10 \%$ for $m_{0}=\mathcal{O}\left(10^{3}\right) \mathrm{TeV}$. 


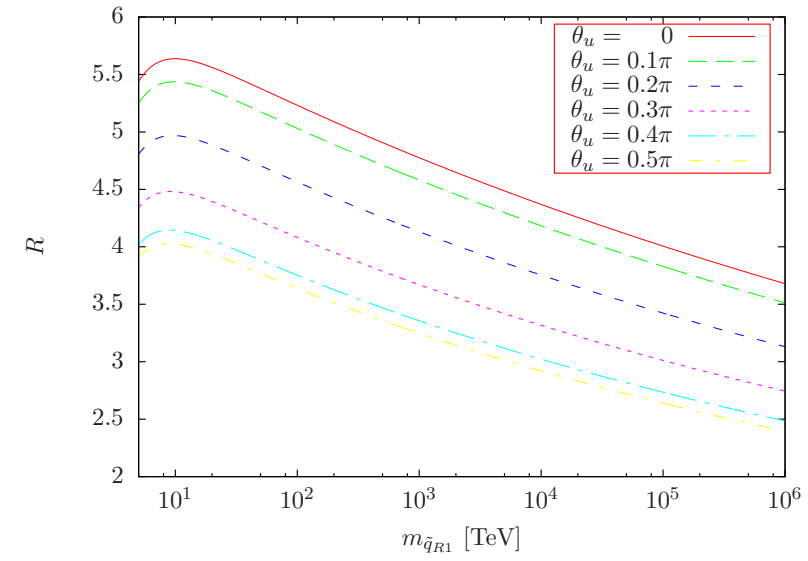

(a) Normalized decay length

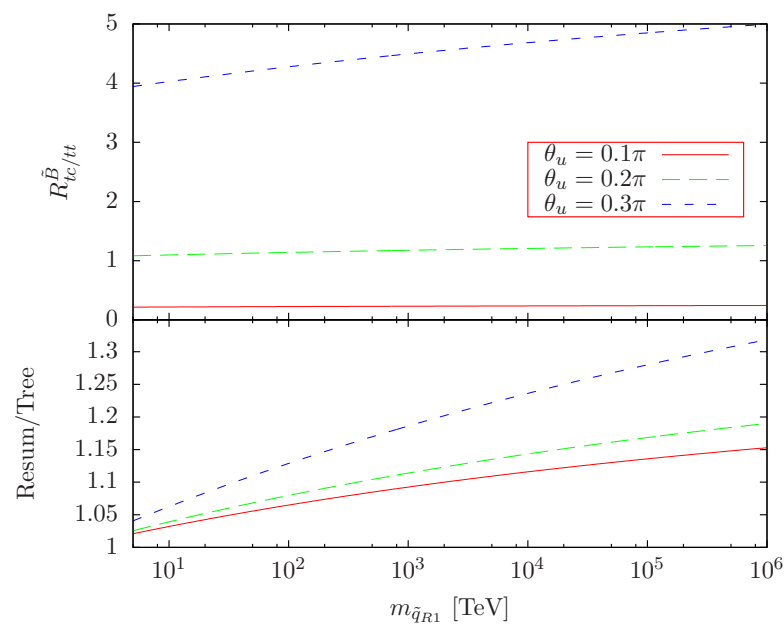

(b) Branching ratio to $t \bar{t}$

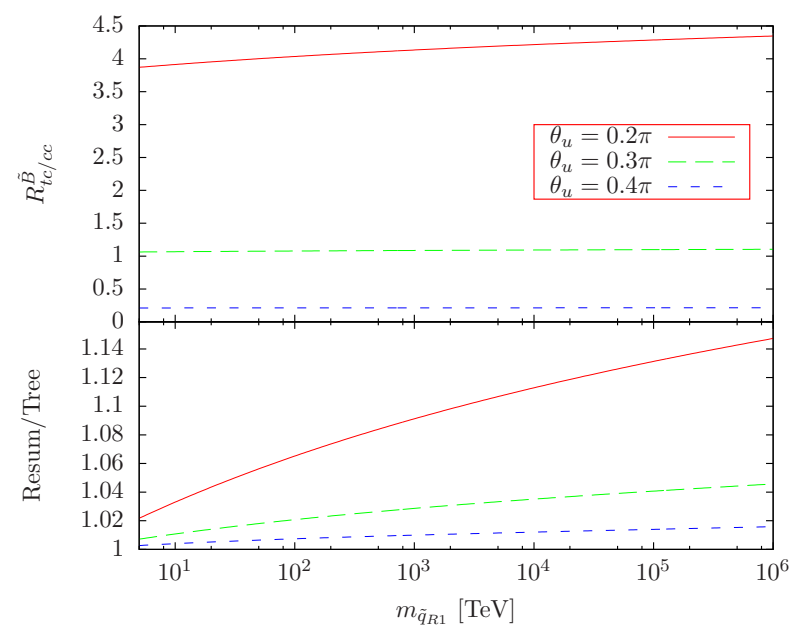

(b) Branching ratio to $c \bar{c}$

Figure 5: Normalized decay length which is defined as $R=\left(c \tau_{\tilde{g}} / 1 \mathrm{~cm}\right)\left(m_{0} / 1000 \mathrm{TeV}\right)^{4}$ (a), branching ratio $R_{t c / t t}^{\tilde{B}}$ (b) and $R_{t c / c c}^{\tilde{B}}$ (c) in a case of light $\tilde{u}_{R}$. We take the gaugino masses as $m_{\tilde{W}}=200 \mathrm{GeV}, m_{\tilde{B}}=400 \mathrm{GeV}$ and $m_{\tilde{g}}=-1.5 \mathrm{TeV}$. 

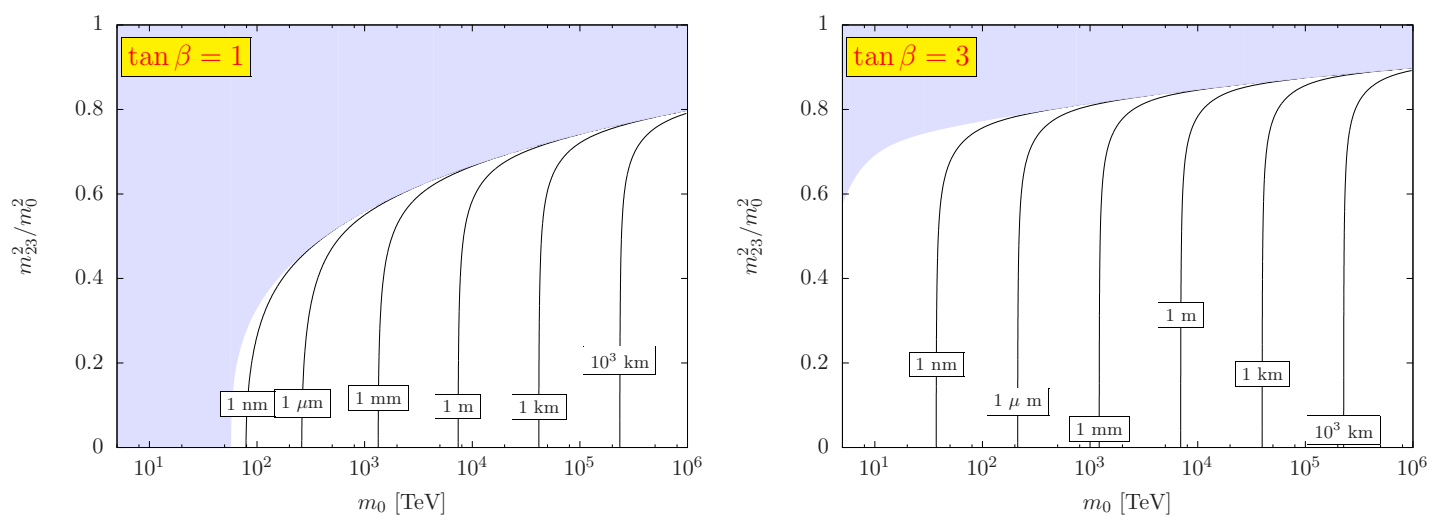

(a) Decay length
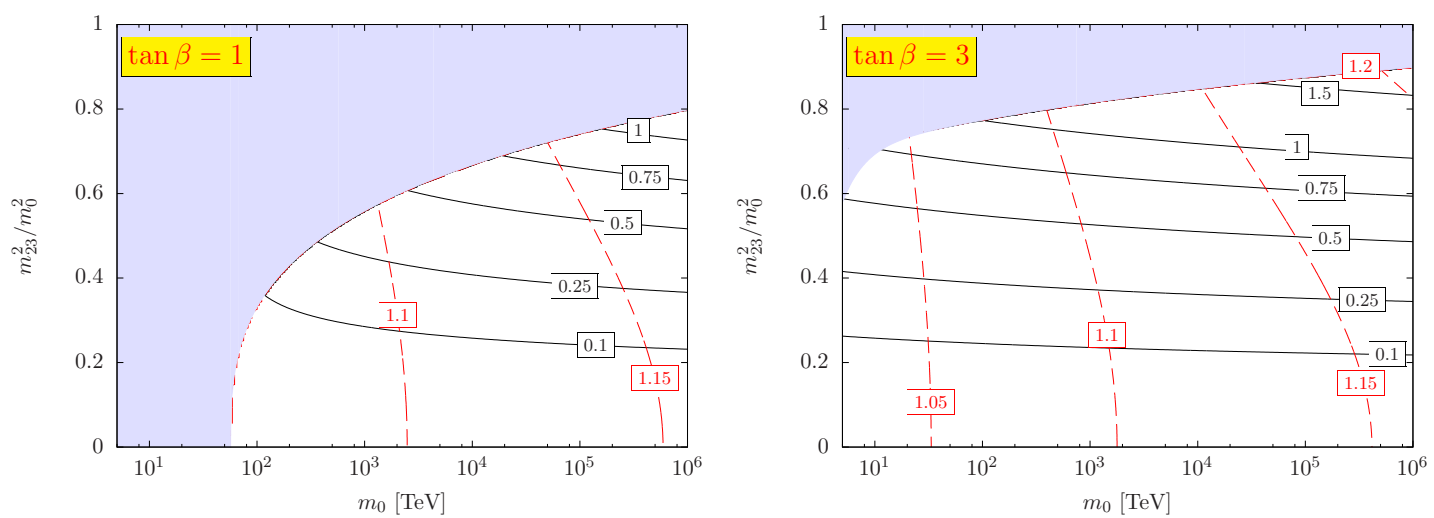

(b) Branching ratio to $t \bar{t}$
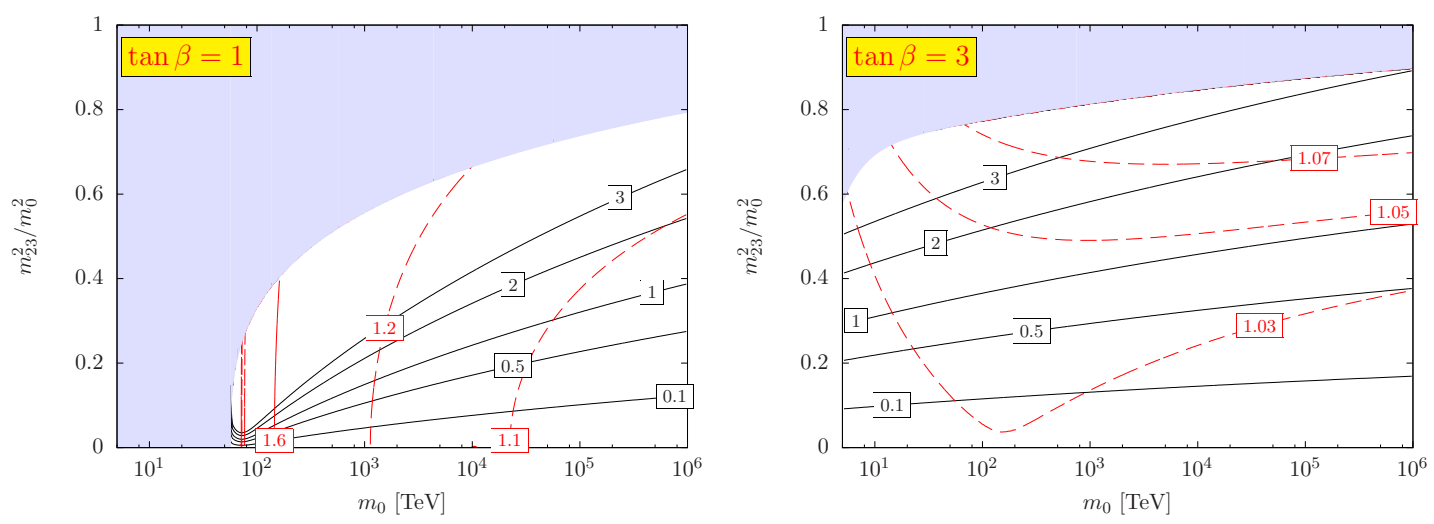

(c) Branching ratio to $c \bar{c}$

Figure 6: Decay length $c \tau_{\tilde{g}}(\mathrm{a})$, Branching fraction $R_{t c / t t}^{\tilde{B}}(\mathrm{~b})$ and $R_{t c / c c}^{\tilde{B}}(\mathrm{c})$ in mSUGRAlike case. In shaded region of the above figures, the lightest squark becomes lighter than the gluino or a tachyon. In figures (b) and (c), black lines show branching ratio $R_{t c / t t}^{\tilde{B}}$ and $R_{t c / c c}^{\tilde{B}}$, respectively, and red-dashed lines shows each $R_{\text {resum }} / R_{\text {tree }}$. 


\section{Low-energy Flavor Constraints and Gluino Decay}

As we have discussed above, the gluino decay directly reflects the structure of the squark sector. For example, the flavor violation of the squark sector leads the flavor violating gluino decay, such as $\tilde{g} \rightarrow t c \tilde{\chi}$. However, in general, the gluino-squark interactions which control the gluino decay, also contribute to low-energy rare processes such as meson mixings and electric dipole moments (EDM's). Therefore, observations of the low-energy physics give constraints on the gluino decay process. In this section, we study the correlation between the low-energy flavor/CP observation and the gluino decay.

\subsection{Flavor/CP Constraints}

In general, SSMs can give visible contributions to low-energy flavor/CP violating processes, even if the SUSY breaking scale is high, say, higher than $\mathcal{O}(10) \mathrm{TeV}$. Here we discuss some low-energy observables, which can give strong constraints on the high-scale SUSY models. For reviews, see, e.g., Refs. [38, 39].

Here we adopt the super-CKM basis (see Appendix A for the correspondence to the weak-basis). We consider the following squark mass-squared matrices in this basis:

$$
m_{a}^{2}=m_{0}^{2}\left(\begin{array}{ccc}
1+\Delta_{1}^{a} & \delta_{12}^{a} & \delta_{13}^{a} \\
\delta_{12}^{a *} & 1+\Delta_{2}^{a} & \delta_{23}^{a} \\
\delta_{13}^{a *} & \delta_{23}^{*} & 1+\Delta_{3}^{a}
\end{array}\right)
$$

where $a=\tilde{Q}_{L}\left(\tilde{d}_{L}\right), \tilde{u}_{R}, \tilde{d}_{R}$. The mass-squared matrix for $\tilde{u}_{L}$ is obtained by $\hat{m}_{\tilde{u}_{L}}^{2}=$ $V_{\mathrm{CKM}} m_{\tilde{Q}_{L}}^{2} V_{\mathrm{CKM}}^{\dagger}$. For the assumption of some charge of the SUSY breaking field, we expect the sizes of $A$-terms are order of the gaugino masses and neglect them. We discuss the current constraints on the flavor violating mass terms.

\section{$\Delta F=2$ process}

One of the strongest constraints on $\delta$ 's comes from $\Delta F=2$ meson mixings. Squarks contribute to the low-energy $\Delta F=2$ operators via loop processes (Fig. 7-(a)). We consider only gluino mediated box diagrams, which give leading contributions. The $K^{0}-$ $\bar{K}^{0}$ system is well measured and gives the strongest constraint on the first and second generation flavor violation. This can constrain $\delta_{12}^{\tilde{Q}_{L}}$ and $\delta_{12}^{\tilde{d}_{R}}$, since they induce direct $s-d$ 


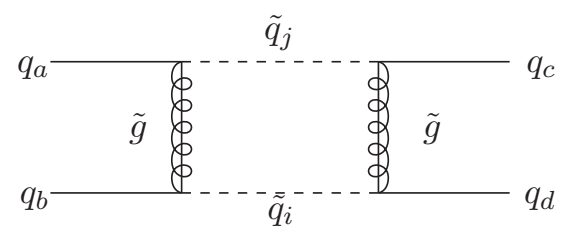

(a) Meson mixing

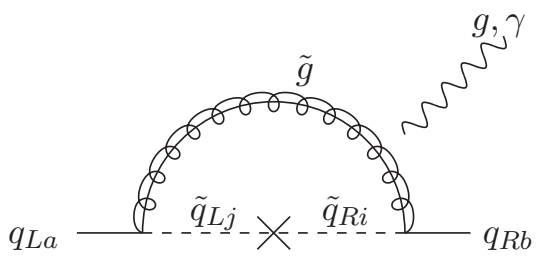

(b) Dipole

Figure 7: Examples of diagrams relevant to low-energy constraints.

mixings. Especially, simultaneous existences of $\delta_{12}^{\tilde{Q}_{L}}, \delta_{12}^{\tilde{d}_{R}}$ and CP-phase are drastically constrained. In addition, it can also constrain products of $\delta_{13}$ and $\delta_{23}$. This is because a squark transition $(1 \rightarrow 3 \rightarrow 2)$ induces large $s-d$ transition.

\section{$\Delta F=1$ process}

The operators with $\Delta F=1$ induce $\Delta F=1$ heavy quark decays, such as $b \rightarrow s \gamma$. These operators are chirality-flipping, and thus the amplitudes of these processes depend on the size of $\mu$ term. If the absolute value of $\mu$ is as much as the squark scale, the observation from $b \rightarrow s \gamma$ provides a strong constraint on the entry of 3-2 element of the squark mass-squared matrices. In the case of $\mathcal{O}(1)$ of $\delta^{23}, m_{0}<\mathcal{O}(10) \mathrm{TeV}$ is excluded.

\section{$\Delta F=0$ process}

Electric and chromo-electric dipole moments (EDM's and CEDM's) of quarks give constraints on the sfermion sector. Although the EDM and CEDM are not flavor violating processes, it can severely constrain the squark flavor violating structure, if both left and right-handed squarks sector have flavor/CP violations and the size of $\mu$ is large. These operators are chirality-flipping and the coefficients are suppressed by the SM fermion masses. When the squark mass-squared matrix is flavor diagonal, these amplitudes are suppressed by the light quark mass $\left(m_{u}, m_{d}\right.$ and $\left.m_{s}\right)$. However once one allows large flavor violation in the squark sector, the suppression is replaced with heavy quarks $\left(m_{t}\right.$ and $m_{b}$ ) and the size of EDM enhanced by $m_{t, b} / m_{u, d, s}$. With $\mathcal{O}(1)$ flavor violation, even PeV-scale SUSY can provide significant EDM signatures as emphasized in Ref. [40]. 


\section{Current Constraints}

In Fig. 8, we show the constraints on some combinations of $\delta$ 's. $\Delta$ 's and $\delta$ 's which are not displayed in the figure are set to be zero. We set the Higgsino and gauginos masses as $\mu=m_{0}, m_{\tilde{g}}=-1.5 \mathrm{TeV}, m_{\tilde{B}}=+400 \mathrm{GeV} m_{\tilde{W}}=+200 \mathrm{GeV}$. Here we set $\tan \beta$ to realize the $125 \mathrm{GeV}$ Higgs mass. In this estimation, we neglect the flavor violation terms and assume that all the SUSY scalar particles have a common mass $m_{0}$ and that $m_{t}=173.2 \mathrm{GeV} .{ }^{6}$ In this setup, for $m_{0} \lesssim 10 \mathrm{TeV}$ or $m_{0} \gtrsim 10^{4} \mathrm{TeV}$, no $\tan \beta$ for the 125 $\mathrm{GeV}$ Higgs mass is found within the range of 1 to 50 . In such cases, we set $\tan \beta=50$ and 1 , respectively. We also require the lightest squark is heavier than the gluino.

To get the constraints, we evaluate the one-loop box and dipole diagrams, evolve the Wilson coefficients down to relevant hadronic scales via RG equations from the QCD interaction and estimate the hadronic matrix elements. For the meson mixings, we use the results of new physics fits of Refs. $[41,42,43]$ and obtain the constraints. The constraints from $K^{0}-\bar{K}^{0}$ and $D^{0}-\bar{D}^{0}$ mixings have large uncertainties, for lack of concrete SM predictions. In our analysis, we assume that the uncertainties of the SM predictions are same as experimental observations, e.g., $\delta\left(\Delta m_{K}\right)_{\mathrm{SM}}=\left(\Delta m_{K}\right)_{\exp }$. Although the hadronic EDMs suffer from large uncertainties, we adopt the result of Ref. [44] and compare them with the current experimental constraints $[45,46]$.

Fig. 8-(a) shows the case of flavor violation of right-handed squarks without CPviolation. Note that the constraints from $K^{0}-\bar{K}^{0}$ and $D^{0}-\bar{D}^{0}$ suffer from large ambiguities, depending on the treatment of the SM predictions. Fig. 8-(b) shows the case of flavor violation of both left and right-handed squarks without CP-violation. Generally, compared with the case of (a), the constraints get severer. Fig. 8-(c) shows the case of flavor violation of only right-handed squarks with $\mathrm{CP}$-violation. We choose the CP-phase so that the strongest constraint can be obtained. In this case, the constraint from $\epsilon_{K}$ is strong and robust. Fig. 8-(d) shows the case of both left and right-handed flavor violation and CP-violation. In this case, we see that even PeV-scale SUSY suffers from strong

\footnotetext{
${ }^{6}$ With large flavor violating mass terms, the mass of the lightest squark can be much less than $m_{0}$ and $\mu$. In this case, large finite corrections can be expected and change the prediction of the Higgs mass. In addition, if $|\mu| \gg m_{\text {squark }}$, the electroweak breaking vacuum may become metastable or unstable. In this paper, we neglect these effects for simplicity.
} 
constraints from the $K^{0}-\bar{K}^{0}$ mixing. Here we show that the EDMs also give powerful constraints on the flavor violation. However note that these constraints strongly depend on the size of $\mu, \tan \beta$ and hadronic uncertainties.

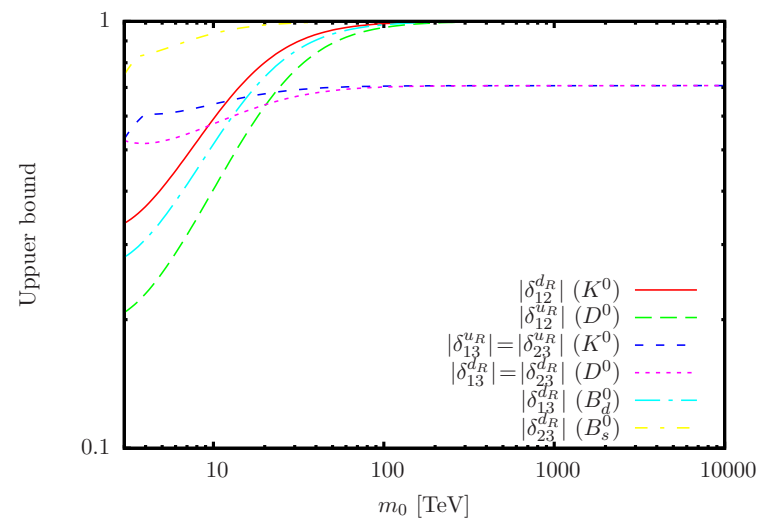

(a) $\mathrm{CP}+\mathrm{R}$

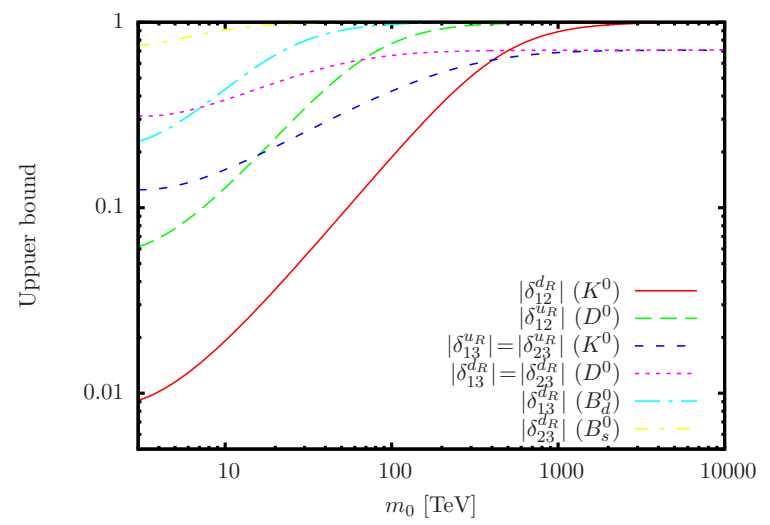

(c) $\mathrm{CPV}+\mathrm{R}$

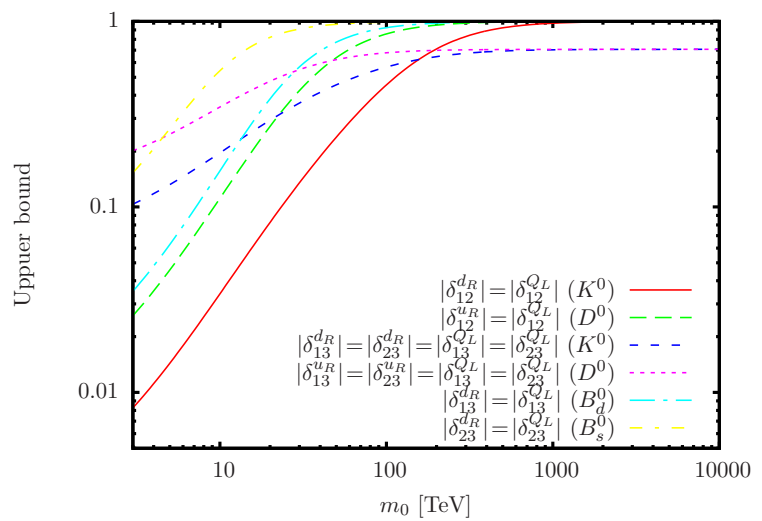

(b) $\mathrm{CP}+\mathrm{LR}$

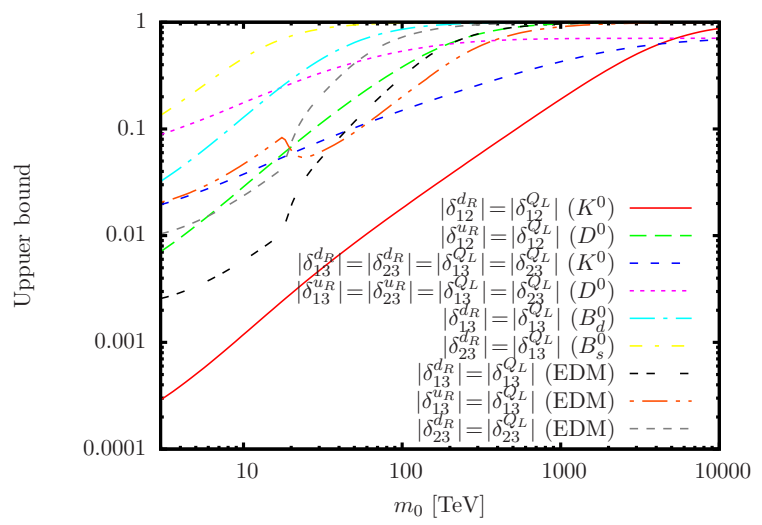

(d) $\mathrm{CPV}+\mathrm{LR}$

Figure 8: Upper-bound on the flavor violating mass terms $\delta$. See text for explanation.

\subsection{Flavorful Gluino Decay}

As discussed in Section 2, the flavor violating gluino decay can be expected for the squark flavor violation. As in Fig. 8, if the squark mass is larger than $\mathcal{O}(100) \mathrm{TeV}$, large flavor violation is allowed from the current constraints. Generally, there are gluino decays $\left(\tilde{g} \rightarrow q_{1} q_{2} \chi\right)$ violating both the first and second flavor violation which comes from either minimal or non-minimal flavor violation. However, instead, we focus on the gluino decays 
accompanied with third flavor violation $\left(\tilde{g} \rightarrow q_{1,2} q_{3} \chi\right)$, since these constraints are weak and third-family violating gluino decay may be experimentally more viable to be discovered.

Using the same setup in Fig. 8, we show the upper-bound on the branching fraction of $\tilde{g} \rightarrow q_{1,2} q_{3} \chi$, keeping the current experimental constraints in Fig. 9. We adopt the lightest squark mass as the boundary scale $\tilde{m}$. As the squark mass scale $m_{0}$ gets larger, the larger flavor violation $\delta$ 's are allowed. In the case of the large $\delta$ 's, the lightest squarks, which are mixed states of different generations, dominate the gluino decay process and the flavor violating branching fraction approximates $50 \%$. Except for the constraints from $\epsilon_{K}$, the squark mass scale higher than $\mathcal{O}\left(10^{2-3}\right)$ TeV can saturate the gluino flavor violating branching fraction. As $m_{0}$ is larger, the RG evolution effects get more efficient and logarithmically enhance this fraction.

\section{Collider Signature}

Let us briefly discuss the collider signature of the gluino decay in the high-scale SUSY models. Distinctive features of the gluino decay in the high-scale SUSY models are the longevity of the gluino lifetime and the flavor violating decay modes. The signatures of the long-lived gluinos are often discussed in the high-scale SUSY scenarios and leave unique signatures $[47,48]$. Instead we focus on the signals of the flavorful gluino decay. Depending on the lifetime of the gluino, the way to identify the flavorful gluino decay differs.

Case I. $c \tau_{\tilde{g}} \ll \mathcal{O}(1) \mathrm{mm}\left(\tilde{m}<\mathcal{O}\left(10^{3}\right) \mathrm{TeV}\right)$

In this case, the impact parameters of the gluino decay products are less than the limitation of the LHC detector. Therefore it is difficult to directly measure the gluino lifetime. Instead, in this case, conventional collider techniques such as heavy-flavor quark and lepton tagging, jet reconstructions and so on are available. Depending on the gluino decay mode, distinctive signatures can be expected [49]. Using, for instance, the techniques developed in Ref. [32], we can estimate the gluino branching fractions.

Case II. $\mathcal{O}(1) \mathrm{mm}<c \tau_{\tilde{g}}<\mathcal{O}(10) \mathrm{m}\left(\mathcal{O}\left(10^{3}\right) \mathrm{TeV}<\tilde{m}<\mathcal{O}\left(10^{4}\right) \mathrm{TeV}\right)$

In this case, although the gluino can decay inside the detector, some of traditional 


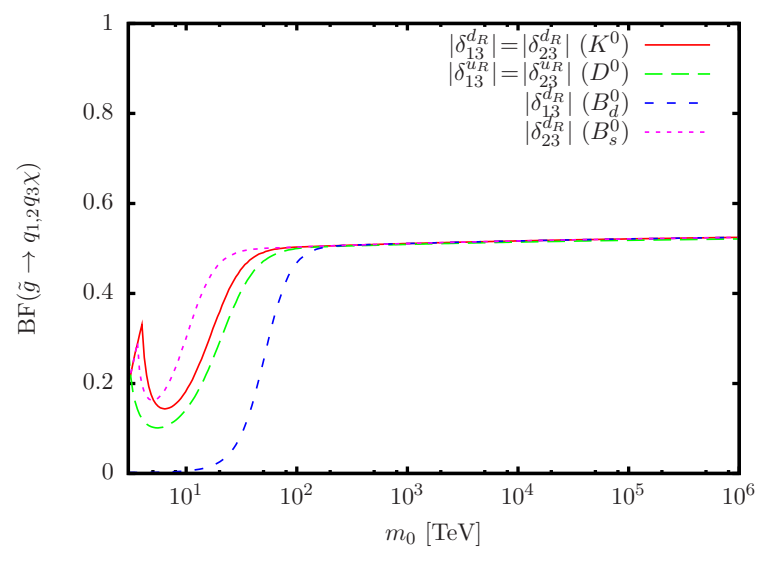

(a) $\mathrm{CP}+\mathrm{R}$

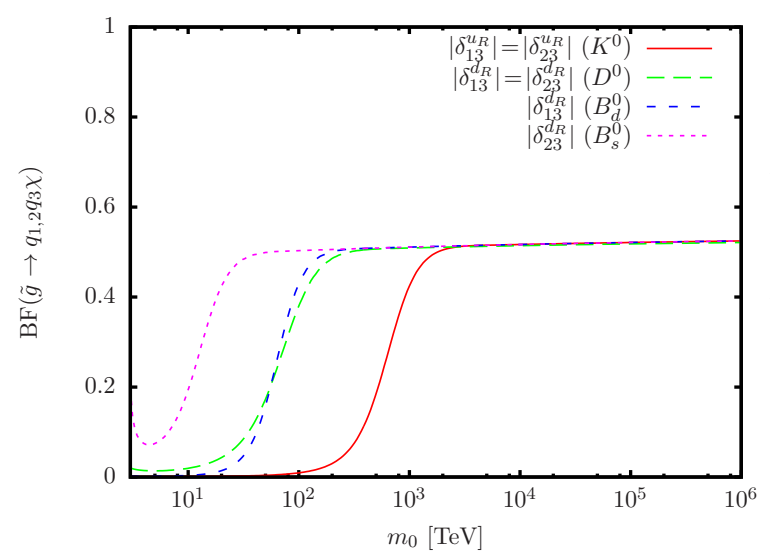

(c) $\mathrm{CPV}+\mathrm{R}$

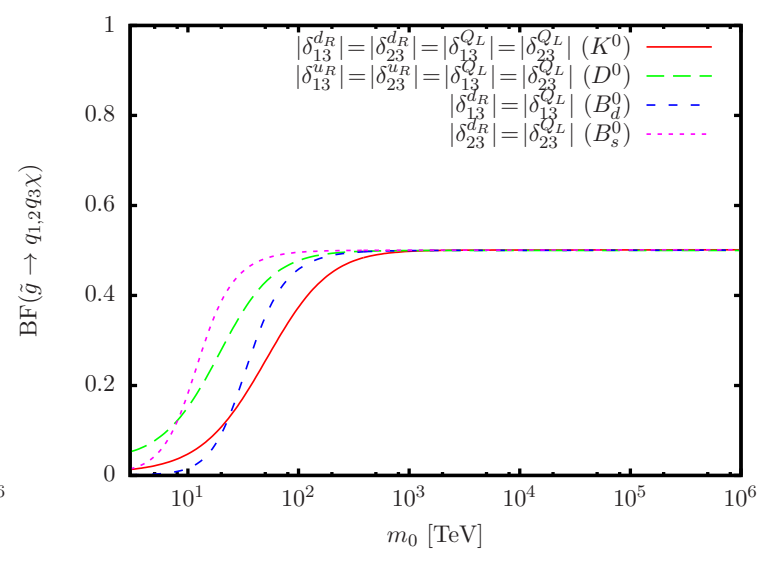

(b) $\mathrm{CP}+\mathrm{LR}$

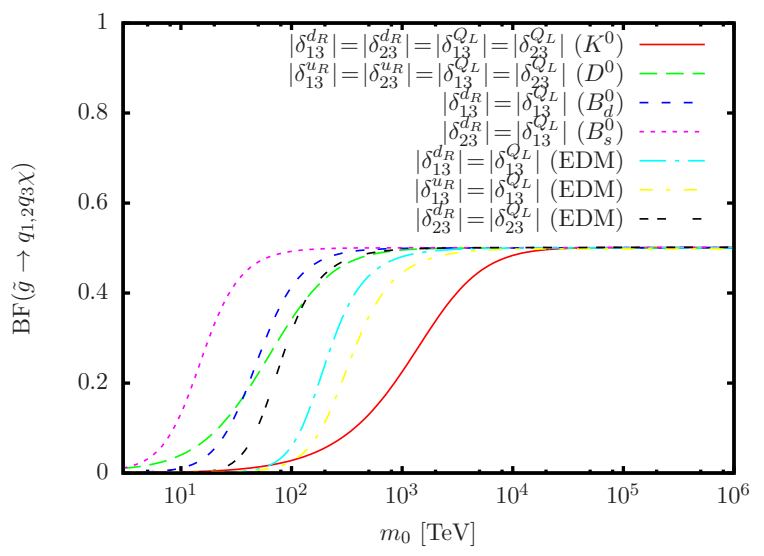

(d) $\mathrm{CPV}+\mathrm{LR}$

Figure 9: Upper-bound on the gluino branching fraction with third-family flavor violation. 
collider techniques will fail. For example, usage of the conventional $b$-tagging is doubtful, since it owes to impact parameters of the $b$ decays. However, studies of collider signatures with displaced vertices are developing. We expect the detailed analysis of track reconstructions and energy deposit in detectors can reveal the displaced gluino decays.

Case III. $c \tau_{\tilde{g}} \gg \mathcal{O}(10) \mathrm{m}\left(\tilde{m} \gg \mathcal{O}\left(10^{4}\right) \mathrm{TeV}\right)$

In this case, the detection of in-flight decays of the gluinos is difficult. However, if a number of gluinos are produced, some of the gluinos can be trapped in the detector [50]. Information of the energy deposit in the gluino decay may be helpful, as discussed in the context of measurement of the branching fraction of the trapped staus [51]. For instance, $t$ quarks from the gluino decay emit leptons, which change hadronic energy deposit of the gluino decay. Therefore a detailed study of the energy deposit of the stopped gluino decay gives us a clue for the gluino decay mode.

Once the gluino decays can be fully identified, ideally, $\mathcal{O}\left(10^{4}\right)$ gluino decays may result in $\mathcal{O}(1) \%$ accuracy of the measurement of the gluino branching fractions. However it is unclear whether we can identify the gluino decay, eliminating the background. The detailed analysis is beyond the scope of this paper and will be done elsewhere [52].

\section{Conclusion and Discussion}

The framework of high-scale SUSY models is well motivated from both phenomenological and theoretical viewpoints. In particular the MSSM with the scalar scale $\mathcal{O}\left(10-10^{6}\right)$ $\mathrm{TeV}$ can explain the Higgs boson with a mass of about $125 \mathrm{GeV}$. In such a framework, while the SUSY particles in the electroweak scale have a rather simple spectrum and can be accessible by experiments, the structure of the sfermion sector has strong model dependence and cannot be directly probed by the experiments. Examining the sfermion sector is essential to discriminate the underlying model. In this paper, we have discussed the gluino decay as a probe of the sfermion sector in a high-scale SUSY model. We have studied the relation between the squark sector and the gluino decay in detail.

If the gaugino mass scale is within the reach of a collider, we can observe the gluino

decay which is strongly affected by the squark sector. The gluino lifetime reflects the 
squark scale and the decay products are sensitive to the squark flavor structure. Therefore the gluino decay will provide us clues to investigate the heavy sfermion sector. Especially, in our study, we focused on the flavor structure of the gluino decay and investigated the correlation with low-energy observations.

The large mass hierarchy between the sfermion and gaugino scale can result in large quantum corrections for the gluino decay. We have used RG methods to tame the quantum corrections. We have found that the squark mass scale is higher than $\mathcal{O}(100) \mathrm{TeV}$, the quantum corrections can affect the branching ratio by $\mathcal{O}(10) \%$, compared to the treelevel results. Ideally, by observing $\mathcal{O}\left(10^{4}\right)$ gluino decays at a collider, we can estimate the branching fraction with $\mathcal{O}(1) \%$ accuracy. Compared to this accuracy, the effects of the quantum corrections are more significant, when we try to reconstruct the squark sector via measurement of the gluino decays.

We also discussed the correlation between the gluino decay and low-energy flavor/CP observations. From the current constraints from the flavor/CP, we have found that the scalar scale $\mathcal{O}\left(10-10^{6}\right) \mathrm{TeV}$ allows large flavor violating gluino decay. Unlike the flavor structure of the gluino decay products, measurement of $\mathrm{CP}$ violation of the gluino decay seems hard. On the other hand, (future) low-energy flavor/CP experiments are sensitive to such CP violation. The combination of the gluino decay, the lifetime and decay modes, and low-energy flavor/CP observations is crucially important for the study of the underlying flavor/CP symmetry of SSMs.

In this study, we focus on the flavor structure of the quark sector. Recently, flavor

violation of a leptonic sector in a high-scale SUSY model is also studied [53]. Combining these sfermion information will provide us a hint for an underlying flavor and GUT model.

\section{Acknowledgements}

SS thanks L. Hall and Y. Nomura for fruitful discussion. The work of RS and KT is supported in part by JSPS Research Fellowships for Young Scientists. This work is also supported by the World Premier International Research Center Initiative (WPI Initiative), MEXT, Japan. 


\section{A Note on Flavor Basis}

In this Appendix, we summarize notations of the flavor structure which is used in this paper. We neglect $A$-terms of squarks, then, the flavor dependent terms in the Lagrangian is written as,

$\mathcal{L}_{\text {Yukawa }}+\mathcal{L}_{\text {soft }}=\left(H Q_{L} Y_{u} u_{R}^{c}+H^{*} Q_{L} Y_{d} d_{R}^{c}+\right.$ h.c. $)-\tilde{Q}_{L}^{*} m_{\tilde{Q}_{L}}^{2} \tilde{Q}_{L}-\tilde{u}_{R}^{c} m_{\tilde{u}_{R}}^{2} \tilde{u}_{R}^{c *}-\tilde{d}_{R}^{c} m_{\tilde{d}_{R}}^{2} \tilde{d}_{R}^{c *}$,

\section{A.1 Weak Basis}

We can redefine quark and squark fields by using $S U(2)_{L} \times U(1)_{Y}$ symmetric redefinition.

$$
\begin{aligned}
Q_{L, i} \rightarrow Q_{L, i}^{\prime}=U_{i j}^{Q} Q_{L, j}, & \tilde{Q}_{L, i} \rightarrow \tilde{Q}_{L, i}^{\prime}=U_{i j}^{Q} \tilde{Q}_{L, j}, \\
u_{R, i}^{c} \rightarrow u_{R, i}^{c}{ }^{\prime}=U_{i j}^{u} u_{L, j}^{c}, & \tilde{u}_{R, i}^{c} \rightarrow u_{R, i}^{{ }^{c}}{ }^{\prime}=U_{i j}^{u} \tilde{u}_{R, j}^{c}, \\
d_{R, i}^{c} \rightarrow d_{R, i}^{c}{ }^{\prime}=U_{i j}^{d} d_{L, j}^{c}, & \tilde{d}_{R, i}^{c} \rightarrow d_{R, i}^{\tilde{c}^{\prime}}=U_{i j}^{d} \tilde{d}_{R, j}^{c} .
\end{aligned}
$$

By using the above redefinition, we take a basis in which Yukawa matrices at electroweak scale is given as,

$$
Y_{u}=V_{\mathrm{CKM}}^{T} \operatorname{diag}\left(y_{u}, y_{c}, y_{t}\right), \quad Y_{d}=\operatorname{diag}\left(y_{d}, y_{s}, y_{b}\right)
$$

Note on that RG evolution of Yukawa matrices destroys the above form except for electroweak scale because we take scale independent $U$ 's. We denote this basis as "weak basis" in this paper. In this basis, we can write interaction terms as $S U(2)_{L}$ symmetric forms, then, this basis is convenient to calculate the $R G$ equations.

\section{A.2 Super-CKM basis}

In this basis, Yukawa interaction of quarks are diagonalized at electroweak scale. Quarks and squarks in this basis are related to ones in weak-basis as,

$$
\begin{array}{llll}
\hat{u}_{L}=V_{\mathrm{CKM}} u_{L}, & \hat{u}_{R}^{c}=u_{R}^{c}, & \hat{d}_{L}=d_{L}, & \hat{d}_{R}^{c}=d_{R}^{c}, \\
\hat{\tilde{u}}_{L}=V_{\mathrm{CKM}} \tilde{u}_{L}, & \hat{\tilde{u}}_{R}^{c}=\tilde{u}_{R}^{c}, & \hat{\tilde{d}}_{L}=\tilde{d}_{L}, & \hat{\tilde{d}}_{R}^{c}=\tilde{d}_{R}^{c} .
\end{array}
$$


Each quark is mass eigenstate, and each squark is defined as a super-partner of each quark. In this basis, mass-squared matrices of squarks are given by,

$$
\mathcal{L}_{\text {soft }}=-\hat{\tilde{u}}_{L}^{*} \hat{m}_{\tilde{u}_{L}}^{2} \hat{\tilde{u}}_{L}-\hat{\tilde{d}}_{L}^{*} \hat{m}_{\tilde{d}_{L}}^{2} \hat{\tilde{d}}_{L}-\hat{\tilde{u}}_{R}^{c} \hat{m}_{\tilde{u}_{R}}^{2} \hat{\tilde{u}}_{R}^{c *}-\hat{\tilde{\tilde{d}}}_{R}^{c} \hat{m}_{\tilde{d}_{R}}^{2} \hat{\tilde{d}}_{R}^{c *}
$$

where,

$$
\hat{m}_{\tilde{u}_{L}}^{2}=V_{\mathrm{CKM}} m_{\tilde{Q}_{L}}^{2} V_{\mathrm{CKM}}^{\dagger}, \quad \hat{m}_{\tilde{d}_{L}}^{2}=m_{\tilde{Q}_{L}}^{2}, \quad \hat{m}_{\tilde{u}_{R}}^{2}=m_{\tilde{u}_{R}}^{2}, \quad \hat{m}_{\tilde{d}_{R}}^{2}=m_{\tilde{d}_{R}}^{2}
$$

\section{B RG Equations for Wilson Coefficients of Higgsinos}

In some high-scale SUSY models, the Higgsino mass is also much smaller than the sfermion and heavy Higgs boson masses. If the gluino is heavier than the Higgsinos, it can decay into the Higgsino. Such decay processes are induced by the following dimension six effective interactions:

$$
\begin{aligned}
Q_{1 u, i j}^{\tilde{H}} & =\left(\tilde{H}_{u} \tilde{g}\right)\left(Q_{L, i} u_{R, j}^{c}\right), \\
Q_{2 u, i j}^{\tilde{H}} & =\left(\tilde{H}_{u} \sigma^{\mu \nu} \tilde{g}\right)\left(Q_{L, i} \sigma_{\mu \nu} u_{R, j}^{c}\right), \\
Q_{5 u}^{\tilde{H}} & =\left(\tilde{H}_{u} \sigma^{\mu \nu} \tilde{g}\right) H G_{\mu \nu}, \\
Q_{1 d, i j}^{\tilde{H}} & =\left(\tilde{H}_{d} \tilde{g}\right)\left(Q_{L, i} d_{R, j}^{c}\right), \\
Q_{2 d, i j}^{\tilde{H}} & =\left(\tilde{H}_{d} \sigma^{\mu \nu} \tilde{g}\right)\left(Q_{L, i} \sigma_{\mu \nu} d_{R, j}^{c}\right), \\
Q_{5 d}^{\tilde{H}} & =\left(\tilde{H}_{d} \sigma^{\mu \nu} \tilde{g}\right) H^{*} G_{\mu \nu} .
\end{aligned}
$$

Dimensionless couplings in MSSM respect PQ-symmetry, then, at the leading order, we do not have to consider PQ-breaking operators, e.g., $\left(\tilde{H}_{d} \tilde{g}\right)\left(q_{L, i} u_{R, j}^{c}\right)^{\dagger}$. The effective Lagrangian contains the following interaction terms:

$$
\begin{gathered}
\mathcal{L}_{\text {eff }}=\frac{1}{\tilde{m}^{2}}\left[\sum_{i, j}\left(C_{1 u, i j}^{\tilde{H}} Q_{1 u, i j}^{\tilde{H}}+C_{2 u, i j}^{\tilde{H}} Q_{2 u, i j}^{\tilde{H}}+C_{1 d, i j}^{\tilde{H}} Q_{1 d, i j}^{\tilde{H}}+C_{2 d, i j}^{\tilde{H}} Q_{2 d, i j}^{\tilde{H}}\right)\right. \\
\left.+C_{5 u}^{\tilde{H}} Q_{5 u}^{\tilde{H}}+C_{5 d}^{\tilde{H}} Q_{5 d}^{\tilde{H}}\right]+ \text { h.c.. }
\end{gathered}
$$


The boundary conditions at the squark mass scale $\tilde{m}$ are given by,

$$
\begin{aligned}
C_{1 u}^{\tilde{H}}(\tilde{m}) & =\frac{g_{s}}{\sqrt{2} \sin \beta} \tilde{m}^{2}\left[\left(m_{\tilde{Q}_{L}}^{2}\right)^{-1} Y_{u}-Y_{u}\left(\left(m_{\tilde{u}_{R}}^{2}\right)^{-1}\right)^{T}\right], \\
C_{2 u}^{\tilde{H}}(\tilde{m}) & =\frac{g_{s}}{4 \sqrt{2} \sin \beta} \tilde{m}^{2}\left[\left(m_{\tilde{Q}_{L}}^{2}\right)^{-1} Y_{u}+Y_{u}\left(\left(m_{\tilde{u}_{R}}^{2}\right)^{-1}\right)^{T}\right], \\
C_{5 u}^{\tilde{H}}(\tilde{m}) & =\frac{g_{s}^{2}}{32 \sqrt{2} \pi^{2} \sin \beta} \tilde{m}^{2}\left(\operatorname{tr}\left[Y_{u} Y_{u}^{\dagger}\left(m_{\tilde{Q}_{L}}^{2}\right)^{-1}\right]+\operatorname{tr}\left[Y_{u}^{*} Y_{u}^{T}\left(m_{\tilde{u}_{R}}^{2}\right)^{-1}\right]\right), \\
C_{1 d}^{\tilde{H}}(\tilde{m}) & =\frac{g_{s}}{\sqrt{2} \cos \beta} \tilde{m}^{2}\left[\left(m_{\tilde{Q}_{L}}^{2}\right)^{-1} Y_{d}-Y_{d}\left(\left(m_{\tilde{d}_{R}}^{2}\right)^{-1}\right)^{T}\right], \\
C_{2 d}^{\tilde{H}}(\tilde{m}) & =-\frac{g_{s}}{4 \sqrt{2} \cos \beta} \tilde{m}^{2}\left[\left(m_{\tilde{Q}_{L}}^{2}\right)^{-1} Y_{d}+Y_{d}\left(\left(m_{\tilde{d}_{R}}^{2}\right)^{-1}\right)^{T}\right], \\
C_{5 d}^{\tilde{H}}(\tilde{m}) & =\frac{g_{s}^{2}}{32 \sqrt{2} \pi^{2} \cos \beta} \tilde{m}^{2}\left(\operatorname{tr}\left[Y_{d} Y_{d}^{\dagger}\left(m_{\tilde{Q}_{L}}^{2}\right)^{-1}\right]+\operatorname{tr}\left[Y_{d}^{*} Y_{d}^{T}\left(m_{\tilde{d}_{R}}^{2}\right)^{-1}\right]\right) .
\end{aligned}
$$

The RG equations for the Wilson coefficients $C^{\tilde{H}}$ 's are given by,

$$
\begin{aligned}
16 \pi^{2} \frac{d C_{1 u}^{\tilde{H}}}{d \log \mu} & =\frac{3 g_{s}^{2}}{N_{C}} C_{1 u}^{\tilde{H}}+\frac{1}{2}\left(Y_{u} Y_{u}^{\dagger}+Y_{d} Y_{d}^{\dagger}\right) C_{1 u}^{\tilde{H}}+C_{1 u}^{\tilde{H}} Y_{u}^{\dagger} Y_{u}, \\
16 \pi^{2} \frac{d C_{2 u}^{\tilde{H}}}{d \log \mu} & =-\left(4 N_{C}+\frac{1}{N_{C}}\right) g_{s}^{2} C_{2 u}^{\tilde{H}}+\frac{1}{2}\left(Y_{u} Y_{u}^{\dagger}+Y_{d} Y_{d}^{\dagger}\right) C_{2 u}^{\tilde{H}}+C_{2 u}^{\tilde{H}} Y_{u}^{\dagger} Y_{u}+2 g_{s} Y_{u} C_{5 u}^{\tilde{H}}, \\
16 \pi^{2} \frac{d C_{5 u}^{\tilde{H}}}{d \log \mu} & =\left(\frac{2}{3} N_{F}-6 N_{C}\right) g_{s}^{2} C_{5 u}^{\tilde{H}}+N_{C} \operatorname{tr}\left[Y_{u} Y_{u}^{\dagger}+Y_{d} Y_{d}^{\dagger}\right] C_{5 u}^{\tilde{H}}+4 g_{s} \operatorname{tr}\left[C_{2 u} Y_{u}^{\dagger}\right] \\
16 \pi^{2} \frac{d C_{1 d}^{\tilde{H}}}{d \log \mu} & =\frac{3 g_{s}^{2}}{N_{C}} C_{1 d}^{\tilde{H}}+\frac{1}{2}\left(Y_{u} Y_{u}^{\dagger}+Y_{d} Y_{d}^{\dagger}\right) C_{1 d}^{\tilde{H}}+C_{1 d}^{\tilde{H}} Y_{d}^{\dagger} Y_{d} \\
16 \pi^{2} \frac{d C_{2 d}^{\tilde{H}}}{d \log \mu} & =-\left(4 N_{C}+\frac{1}{N_{C}}\right) g_{s}^{2} C_{2 d}^{\tilde{H}}+\frac{1}{2}\left(Y_{u} Y_{u}^{\dagger}+Y_{d} Y_{d}^{\dagger}\right) C_{2 d}^{\tilde{H}}+C_{2 d}^{\tilde{H}} Y_{d}^{\dagger} Y_{d}+2 g_{s} Y_{d} C_{5 d}^{\tilde{H}}, \\
16 \pi^{2} \frac{d C_{5 d}^{\tilde{H}}}{d \log \mu} & =\left(\frac{2}{3} N_{F}-6 N_{C}\right) g_{s}^{2} C_{5 d}^{\tilde{H}}+N_{C} \operatorname{tr}\left[Y_{u} Y_{u}^{\dagger}+Y_{d} Y_{d}^{\dagger}\right] C_{5 d}^{\tilde{H}}+4 g_{s} \operatorname{tr}\left[C_{2 d} Y_{d}^{\dagger}\right]
\end{aligned}
$$

Here, we take into account only quark Yukawa and QCD interactions. We have checked Eqs. (81-86) are consistent with Ref. [31] in the flavor-symmetric case. For the RG equations of dimensionless coupling constants, see the Appendix of Ref. [9].

\section{RG Equations of Soft Mass with Flavor Violation}

In this appendix, we show the RG equations for sfermion soft masses. Here, we neglect trilinear-coupling, gaugino masses. We assume relatively small $\tan \beta$, then neglect down- 
type quark and lepton Yukawa couplings.

$$
\begin{aligned}
16 \pi^{2} \frac{d m_{\tilde{Q}_{L}}^{2}}{d \log \mu} & =2\left\{Y_{u}^{*} Y_{u}^{T}, m_{\tilde{Q}_{L}}^{2}\right\}+2 Y_{u}^{*}\left(m_{\tilde{u}_{R}}^{2 T}+m_{H_{u}}^{2} \mathbf{1}\right) Y_{u}^{T}+\frac{g_{1}^{2} S}{5} \mathbf{1}, \\
16 \pi^{2} \frac{d m_{\tilde{u}_{R}}^{2}}{d \log \mu} & =4\left\{Y_{u}^{\dagger} Y_{u}, m_{\tilde{u}_{R}}^{2}\right\}+4 Y_{u}^{\dagger}\left(m_{\tilde{Q}_{L}}^{2 T}+m_{H_{u}}^{2} \mathbf{1}\right) Y_{u}-\frac{4 g_{1}^{2} S}{5} \mathbf{1} \\
16 \pi^{2} \frac{d m_{\tilde{d}_{R}}^{2}}{d \log \mu} & =\frac{2 g_{1}^{2} S}{5} \mathbf{1} \\
16 \pi^{2} \frac{d m_{\tilde{L}_{L}}^{2}}{d \log \mu} & =-\frac{3 g_{1}^{2} S}{5} \mathbf{1} \\
16 \pi^{2} \frac{d m_{\tilde{e}_{R}}^{2}}{d \log \mu} & =\frac{6 g_{1}^{2} S}{5} \mathbf{1} \\
16 \pi^{2} \frac{d m_{H_{u}}^{2}}{d \log \mu} & =6 \operatorname{tr}\left[Y_{u}^{*} Y_{u}^{T} m_{\tilde{Q}_{L}}^{2}\right]+6 \operatorname{tr}\left[Y_{u}^{\dagger} Y_{u} m_{\tilde{u}_{R}}^{2}\right]+6 \operatorname{tr}\left[Y_{u} Y_{u}^{\dagger}\right] m_{H_{u}}^{2}+\frac{3 g_{1}^{2} S}{5} \\
16 \pi^{2} \frac{d m_{H_{d}}^{2}}{d \log \mu} & =-\frac{3 g_{1}^{2} S}{5} .
\end{aligned}
$$

Here, sfermion mass-squared matrices $m_{\tilde{Q}_{L}}^{2}, m_{\tilde{u}_{R}}^{2}, m_{\tilde{d}_{R}}^{2}, m_{\tilde{L}_{L}}^{2}$ and $m_{\tilde{e}_{R}}^{2}$ and Yukawa matrix $Y_{u}$ are $3 \times 3$ matrices in flavor space. $S=m_{H_{u}}^{2}-m_{H_{d}}^{2}+\operatorname{tr}\left[m_{Q}^{2}-2 m_{u}^{2}+m_{d}^{2}-m_{L}^{2}+m_{e}^{2}\right]$. $\{A, B\}=A B+B A, \mathbf{1}=\operatorname{diag}(1,1,1)$.

\section{References}

[1] G. Aad et al. [ATLAS Collaboration], Phys. Lett. B 716, 1 (2012) [arXiv:1207.7214 [hep-ex]].

[2] S. Chatrchyan et al. [CMS Collaboration], Phys. Lett. B 716, 30 (2012) [arXiv:1207.7235 [hep-ex]].

[3] J. D. Wells, hep-ph/0306127.

[4] N. Arkani-Hamed and S. Dimopoulos, JHEP 0506, 073 (2005) [hep-th/0405159].

[5] G. F. Giudice and A. Romanino, Nucl. Phys. B 699, 65 (2004) [Erratum-ibid. B 706, 65 (2005)] [hep-ph/0406088].

[6] N. Arkani-Hamed, S. Dimopoulos, G. F. Giudice and A. Romanino, Nucl. Phys. B 709, 3 (2005) [hep-ph/0409232].

[7] J. D. Wells, Phys. Rev. D 71, 015013 (2005) [hep-ph/0411041]. 
[8] Y. Okada, M. Yamaguchi and T. Yanagida, Prog. Theor. Phys. 85, 1 (1991); Y. Okada, M. Yamaguchi and T. Yanagida, Phys. Lett. B 262, 54 (1991); J. R. Ellis, G. Ridolfi and F. Zwirner, Phys. Lett. B 257, 83 (1991); H. E. Haber and R. Hempfling, Phys. Rev. Lett. 66, 1815 (1991); H. E. Haber and R. Hempfling, Phys. Rev. Lett. 66, 1815 (1991); J. R. Ellis, G. Ridolfi and F. Zwirner, Phys. Lett. B 262, 477 (1991).

[9] G. F. Giudice and A. Strumia, Nucl. Phys. B 858, 63 (2012) [arXiv:1108.6077 [hep$\mathrm{ph}]]$.

[10] G. F. Giudice, M. A. Luty, H. Murayama and R. Rattazzi, JHEP 9812, 027 (1998) [hep-ph/9810442]; L. Randall and R. Sundrum, Nucl. Phys. B 557, 79 (1999) [hep-th/9810155]; M. Dine and D. MacIntire, Phys. Rev. D 46, 2594 (1992) [hepph/9205227]; J. A. Bagger, T. Moroi and E. Poppitz, JHEP 0004, 009 (2000) [hepth/9911029]; P. Binetruy, M. K. Gaillard and B. D. Nelson, Nucl. Phys. B 604, 32 (2001) [hep-ph/0011081].

[11] L. J. Hall and Y. Nomura, JHEP 1201, 082 (2012) [arXiv:1111.4519 [hep-ph]]; L. J. Hall, Y. Nomura and S. Shirai, JHEP 1301, 036 (2013) [arXiv:1210.2395 [hep$\mathrm{ph}]]$.

[12] M. Ibe and T. T. Yanagida, Phys. Lett. B 709, 374 (2012) [arXiv:1112.2462 [hepph]]; M. Ibe, S. Matsumoto and T. T. Yanagida, Phys. Rev. D 85, 095011 (2012) [arXiv:1202.2253 [hep-ph]].

[13] A. Arvanitaki, N. Craig, S. Dimopoulos and G. Villadoro, JHEP 1302, 126 (2013) [arXiv:1210.0555 [hep-ph]].

[14] N. Arkani-Hamed, A. Gupta, D. E. Kaplan, N. Weiner and T. Zorawski, arXiv:1212.6971 [hep-ph].

[15] M. Ibe, T. Moroi and T. T. Yanagida, Phys. Lett. B 644, 355 (2007) [hepph/0610277]; M. R. Buckley, L. Randall and B. Shuve, JHEP 05, 097 (2011) [arXiv:0909.4549 [hep-ph]].

[16] S. Asai, T. Moroi, K. Nishihara and T. T. Yanagida, Phys. Lett. B 653, 81 (2007) [arXiv:0705.3086 [hep-ph]]; S. Asai, T. Moroi and T. T. Yanagida, Phys. Lett. B 664, 
185 (2008) [arXiv:0802.3725 [hep-ph]]; S. Asai, Y. Azuma, O. Jinnouchi, T. Moroi, S. Shirai and T. T. Yanagida, Phys. Lett. B 672, 339 (2009) [arXiv:0807.4987 [hep$\mathrm{ph}]]$.

[17] T. Gherghetta, G. F. Giudice and J. D. Wells, Nucl. Phys. B 559, 27 (1999) [hep-ph/9904378]; T. Moroi and L. Randall, Nucl. Phys. B 570, 455 (2000) [hep$\mathrm{ph} / 9906527]$.

[18] J. Hisano, S. Matsumoto, O. Saito and M. Senami, Phys. Rev. D 73, 055004 (2006) [hep-ph/0511118]; J. Hisano, S. Matsumoto, M. Nagai, O. Saito and M. Senami, Phys. Lett. B 646, 34 (2007) [hep-ph/0610249].

[19] J. Hisano, K. Ishiwata and N. Nagata, Phys. Rev. D 82, 115007 (2010) [arXiv:1007.2601 [hep-ph]]; J. Hisano, K. Ishiwata, N. Nagata and T. Takesako, JHEP 07, 005 (2011) [arXiv:1104.0228 [hep-ph]]; R. J. Hill and M. P. Solon, Phys. Lett. B 707, 539 (2012) [arXiv:1111.0016 [hep-ph]]; J. Hisano, K. Ishiwata and N. Nagata, Phys. Rev. D 87, 035020 (2013) [arXiv:1210.5985 [hep-ph]].

[20] J. Hisano, S. .Matsumoto, M. M. Nojiri and O. Saito, Phys. Rev. D 71, 063528 (2005) [hep-ph/0412403].

[21] T. Moroi and K. Nakayama, Phys. Lett. B 710, 159 (2012) [arXiv:1112.3123 [hep$\mathrm{ph}]]$.

[22] T. Cohen, M. Lisanti, A. Pierce and T. R. Slatyer, arXiv:1307.4082 [hep-ph].

[23] J. Fan and M. Reece, arXiv:1307.4400 [hep-ph].

[24] K. I. Izawa, Y. Nomura, K. Tobe and T. Yanagida, Phys. Rev. D 56, 2886 (1997) [hep-ph/9705228];

[25] Y. Nomura and K. Tobe, Phys. Rev. D 58, 055002 (1998) [hep-ph/9708377].

[26] Z. Komargodski and D. Shih, JHEP 0904, 093 (2009) [arXiv:0902.0030 [hep-th]].

[27] S. Shirai, M. Yamazaki and K. Yonekura, JHEP 1006, 056 (2010) [arXiv:1003.3155 [hep-ph]].

[28] M. Ibe and R. Sato, Phys. Lett. B 717, 197 (2012) [arXiv:1204.3499 [hep-ph]].

[29] R. Saito and S. Shirai, Phys. Lett. B 713, 237 (2012) [arXiv:1201.6589 [hep-ph]]. 
[30] M. Toharia and J. D. Wells, JHEP 0602, 015 (2006) [hep-ph/0503175].

[31] P. Gambino, G. F. Giudice and P. Slavich, Nucl. Phys. B 726, 35 (2005) [hep$\mathrm{ph} / 0506214]$.

[32] R. Sato, S. Shirai and K. Tobioka, JHEP 1211, 041 (2012) [arXiv:1207.3608 [hep$\mathrm{ph}]]$.

[33] A. Arvanitaki, C. Davis, P. W. Graham, A. Pierce and J. G. Wacker, Phys. Rev. D 72, 075011 (2005) [hep-ph/0504210].

[34] M. E. Machacek and M. T. Vaughn, Phys. Lett. B 103, 427 (1981).

[35] M. Ibe, S. Matsumoto and R. Sato, Phys. Lett. B 721, 252 (2013) [arXiv:1212.5989 [hep-ph]].

[36] ATLAS collaboration, ATLAS-CONF-2013-069

[37] R. Barbieri, G. Gamberini, G. F. Giudice and G. Ridolfi, Nucl. Phys. B 301, 15 (1988);

[38] F. Gabbiani, E. Gabrielli, A. Masiero and L. Silvestrini, Nucl. Phys. B 477, 321 (1996) [hep-ph/9604387].

[39] W. Altmannshofer, A. J. Buras, S. Gori, P. Paradisi and D. M. Straub, Nucl. Phys. B 830, 17 (2010) [arXiv:0909.1333 [hep-ph]].

[40] D. McKeen, M. Pospelov and A. Ritz, arXiv:1303.1172 [hep-ph].

[41] M. Bona et al. [UTfit Collaboration], JHEP 0803, 049 (2008) [arXiv:0707.0636 [hep$\mathrm{ph}]]$.

[42] A. J. Bevan et al. [UTfit Collaboration], JHEP 1210, 068 (2012) [arXiv:1206.6245 [hep-ph]].

[43] http://www.utfit.org/UTfit/ResultsWinter2013PreMoriond

[44] J. Hisano and Y. Shimizu, Phys. Rev. D 70, 093001 (2004) [hep-ph/0406091].

[45] C. A. Baker, D. D. Doyle, P. Geltenbort, K. Green, M. G. D. van der Grinten, P. G. Harris, P. Iaydjiev and S. N. Ivanov et al., Phys. Rev. Lett. 97, 131801 (2006) [hep-ex/0602020]. 
[46] W. C. Griffith, M. D. Swallows, T. H. Loftus, M. V. Romalis, B. R. Heckel and E. N. Fortson, Phys. Rev. Lett. 102, 101601 (2009).

[47] J. L. Hewett, B. Lillie, M. Masip and T. G. Rizzo, JHEP 0409, 070 (2004) [hepph/0408248].

[48] K. Cheung and W. -Y. Keung, Phys. Rev. D 71, 015015 (2005) [hep-ph/0408335].

[49] A. Bartl, H. Eberl, E. Ginina, B. Herrmann, K. Hidaka, W. Majerotto and W. Porod, Phys. Rev. D 84, 115026 (2011) [arXiv:1107.2775 [hep-ph]].

[50] A. Arvanitaki, S. Dimopoulos, A. Pierce, S. Rajendran and J. G. Wacker, Phys. Rev. D 76, 055007 (2007) [hep-ph/0506242].

[51] T. Ito, K. Nakaji and S. Shirai, Phys. Lett. B 706, 314 (2012) [arXiv:1104.2101 [hep-ph]].

[52] R. Sato, S. Shirai and K. Tobioka, in preparation.

[53] T. Moroi and M. Nagai, Phys. Lett. B 723, 107 (2013) [arXiv:1303.0668 [hep-ph]], T. Moroi, M. Nagai and T. T. Yanagida, arXiv:1305.7357 [hep-ph]. 